\title{
Rechtsextreme Muslimhetze: Die Instrumentalisierung von Religion als Vote-Seeking-Strategie der AfD
}

\author{
Christoph Bitzl (D) - Michael Kurze
}

Eingegangen: 2. Februar 2021 / Überarbeitet: 29. Juli 2021 / Angenommen: 12. August 2021 / Online publiziert: 27. September 2021

(C) Der/die Autor(en) 2021

Zusammenfassung Die AfD setzt in ihrer Identitätskonstruktion zunehmend auf einen populistischen Gegensatz zwischen „,christlich-jüdischem Abendland“ und „,dem Islam“, obwohl sie sich weder durch eine besondere Nähe zur Kirche und christlichen Wähler:innen noch zur jüdischen Gemeinde auszeichnet. Der folgende Beitrag zeigt anhand einer Analyse von Programmen, Äußerungen und weiteren Veröffentlichungen, dass sich die Partei einer bestehenden Muslimfeindlichkeit in der Bevölkerung bedient und Religion zum Zweck der nativistischen Mobilisierung und Stimmenmaximierung instrumentalisiert. Sowohl der Islam als auch Christentum und Judentum werden zum Träger inhärenter kultureller Merkmale umgedeutet und damit von ihrer religiösen Bedeutung losgelöst. Dabei bedient sich die AfD in ihren essenzialisierenden Zuschreibungen einer antimuslimisch-rassistischen Rhetorik. Durch die Einbettung in einen „Kampf der Kulturen“ schafft es die Partei, ihre mitunter rechtsextremen nativistischen Positionen, die sich vor allem in antipluralistischen Haltungen und einer Unterminierung der Religionsfreiheit zeigen, als Verteidigung liberaler christlich-aufgeklärter Werte, gar als Philosemitismus, zu verklausulieren. Der Beitrag zeigt, dass diese Islamisierung der Debatten und die gleichzeitige Berufung auf ein ,christlich-jüdisches“ Erbe einem wahltaktischen Kalkül zur Stimmenmaximierung folgt, das über einen vermeintlich drohenden Identitätsverlust den rechten Rand und die bürgerliche Mitte gleichermaßen inkludiert, während man sich selbst vom Vorwurf des Rechtsextremismus freispricht.

Schlüsselwörter Rechtspopulismus · AfD · Rechtsextremismus ·

Antimuslimischer Rassismus · Vote-Seeking

Christoph Bitzl ( $\bowtie)$

Hochschule für angewandte Wissenschaften Ansbach, Ansbach, Deutschland

E-Mail: christoph.bitzl@hs-ansbach.de

Michael Kurze

Regensburg, Deutschland

E-Mail: m.kurze@gmx.de 


\title{
Anti-Muslim agitation: the instrumentalization of religion as a vote- seeking-strategy by the AfD
}

\begin{abstract}
In recent years the German right-wing populist party AfD (Alternative for Germany) has re-semanticized the term "christliches Abendland" ("Christian Occident") and increasingly refers to its political and cultural values as a "ChristianJewish" heritage. This comes to a surprise since the party is a major critic of the church and its refugee policy and does neither poll a high count of votes from Christian voters nor show a proximity to Judaism and Jewish life in Germany. As this article demonstrates, the AfD uses its religious framing as a strategy to maximize votes. By referring to Christianity and expressing a philosemitic stance, the party emphasizes its refusal of Islam and Muslims in Germany. It harnesses religious framework to underline alleged cultural and political differences between Muslims and the rest of the population. Thus the AfD exploits the general popularity of antiMuslim attitudes in German society in order to promote its extreme right-wing and nativist concepts of policy. Using racist rhetoric and referring to "Christian-Jewish" values, the party constructs a dichotomy of "German" and "Muslim" culture to legitimate its postulations for a stop of migration and restricted rights for Muslims. The paper argues that all this has to be assessed as a vote-seeking-strategy to exploit wide-spread fears of a loss of identity and a nativist resistance towards a pluralist society that increasingly culminate in anti-Muslim sentiments.
\end{abstract}

Keywords Right-wing populism · Alternative für Deutschland · Right-wing extremism $\cdot$ Islamophobia $\cdot$ Vote-seeking

\section{Einleitung}

Das „,christlich-jüdische Abendland“ ist zu einem viel bemühten kulturellen Referenzrahmen rechtspopulistischer Parteien geworden. Akteur:innen wie die Alternative für Deutschland (AfD) oder die Freiheitliche Partei Österreichs (FPÖ) beschwören das „christlich-europäische Erbe“ im Kampf gegen die vermeintliche Bedrohung einer „Islamisierung des Abendlandes“. Diesen zunehmenden Wechsel des Bezugsrahmens von Nationalismus zu einer breiteren christlich-europäischen Tradition bezeichnet Rogers Brubaker (2017, S. 1193) als ,,identitarian ,Christianis$\mathrm{m}$ ““. Abseits dieser Identitätskonstruktion weisen die AfD und ihre Anhänger:innen auf der einen Seite und christliche Akteur:innen auf der anderen Seite jedoch keine besonders großen Schnittmengen in der politischen Themensetzung auf. Zwar bieten das konservative Familienbild und der Einsatz für ein weitreichendes Abtreibungsverbot einen Anknüpfungspunkt für fundamentalisch-christliche Gruppierungen (vgl. Siri 2016, S. 77f), jedoch gehörten die Kirchen sowie kirchliche Gruppen neben den Gemeinden zu den aktivsten Unterstützer:innen von Geflüchteten während der sogenannten „Flüchtlingskrise“ (vgl. Casanova 2020, S. 37), womit sie in diesem Bereich einen klaren Gegenpol zu rechtspopulistischen Parteien bildeten. Unter den Wähler:innen der AfD ist indes weder ein positiver Effekt der Konfessionszugehörigkeit noch eine positive Auswirkung tieferer Religiosität zu erkennen 
(vgl. Pickel 2018, S. 295). Dasselbe gilt für die Bezugnahme auf das Judentum, die sich in der Semantik des ,christlich-jüdischen Abendlandes“, aber auch in israelfreundlichen Statements der Partei zeigt. Dies steht in deutlichem Widerspruch zu geschichtsrevisionistischen und völkischen-nationalistischen Aussagen von AfDParteifunktionär:innen und führt unweigerlich zu der Frage, warum die Alternative für Deutschland an der widersprüchlichen religiösen Identitätskonstruktion des „christlich-jüdischen Abendlandes“ festhält. Zunächst ist in diesem Zusammenhang die Konstruktion eines möglichst exklusiven Gegenpols zu muslimischen Zuwander:innen und Bürger:innen zu nennen. In diesem Beitrag wird darauf basierend die These vertreten, dass sich die AfD die ,Islamisierung“ (Tiesler 2006) öffentlicher Diskurse zu Nutze macht, um vorhandene rechtsextreme Einstellungsmuster in der Bevölkerung zu bedienen und unter dem Deckmantel der liberalen Demokratie Wähler:innenstimmen zu maximieren. Die AfD ist folglich „Christian largely to the extent that they reject Islam“ (Roy 2016, S. 186). Die religiöse Bezugnahme verklausuliert somit nativistische Positionen, um diese gesellschaftlich anschlussfähig zu machen.

Die verengte Überbewertung des religiös konnotierten „Kulturkampfs“ gegen den Islam ist eingebettet in die rechtspopulistische Ideologie der Partei. Aufbauend auf der Herausarbeitung der Grundlagen dieses populistischen und nativistischen Weltbildes wird mit dem Verweis auf die kontinuierliche migrationsabwehrende Haltung im bundesdeutschen Diskurs (vgl. Foroutan 2019; Spielhaus 2013; Morgenstern 2002) empirisch gezeigt, wie sich die AfD muslimfeindliche Einstellungen in weiten Teilen der Bevölkerung zur Bestärkung ihrer eigenen Ideologie zu Nutze macht. Dabei soll neben der Analyse explizit muslim- und islamfeindlicher Aussagen in Programmen, Reden und Aussagen von Funktionär:innen auch die zu diesem Zwecke instrumentalisierende Umwegkommunikation über Christen- und Judentum dargelegt werden. Diese ,instrumentelle Dreiecksbeziehung“ (Keskinkılıç 2019, S. 59) religiöser Bezugnahme, so soll letztlich gezeigt werden, nutzt die AfD vor allem, um ihre nativistische Agenda öffentlich anschlussfähig zu machen und im Sinne des Vote-Seekings (vgl. Müller und Strøm 2010) Wähler:innen zu gewinnen.

\section{Das heartland als imaginierte Volksgemeinschaft der AfD}

Ein rechtspopulistisches Weltbild bildet den Ausgangspunkt der Konstruktion von Freund- und Feindgruppen im Diskurs der AfD und damit die Grundlage ihrer völkisch-nationalistischen Agenda.

Im Anschluss an Frank Decker und Marcel Lewandowsky (2012, S. 271) sowie Cas Mudde (2004, S. 544) wird Populismus hier als „Bindestrich-Ideologie“ bzw. ,thin-centered ideology“ verstanden, der gewisse ideologische Kernbestandteile inhärent sind, wobei offene Fragen durch andere Weltbilder ergänzt werden können und teilweise auch müssen. Im Kern des Populismus steht die Vorstellung eines nahezu unlösbaren Konflikts zwischen einem homogenen Volk und einer korrupten Elite. Dieser Zustand sei durch die angeblich notwendige Wiederherstellung echter Volksherrschaft zu überwinden (vgl. Rydgren 2004, S. 201; Mudde 2004, S. 543; Stanley 2008, S. 102; Canovan 2002, S. 27). Ziel ist die Rückkehr zu einem sta- 
tus-quo-ante, einer imaginierten Zeit vor dem vermeintlichen Machtmissbrauch der Eliten, ,als die Welt noch in Ordnung war“ (vgl. Heinisch und Mazzoleni 2017, S. 109).

Der rechte Populismus definiert sich über eine romantisch überhöhte Darstellung des Volkes, das als homogene Einheit mit einem klar erfassbaren Willen gezeichnet wird, als dessen Sprachrohr die Populist:innen auftreten (vgl. Taggart 2002, S. 96; Spier 2010, S. 20). Um die vermeintliche Einmütigkeit und Homogenität des ethnokulturell verstandenen Volkes glaubhafter erscheinen zu lassen, werden ganze Teile der Bevölkerung von diesem imaginierten Volk ausgeschlossen (vgl. Rydgren 2004, S. 206). So ist das Volk der Populist:innen keinesfalls mit der Bevölkerung eines Staates gleichzusetzen (vgl. Decker 2011, S. 41). Paul Taggart (2004, S. 274) spricht in diesem Zusammenhang vom populistischen heartland, that represents an idealised conception of the community they serve“. Aus diesem hochstilisierten Verständnis eines schwarmintelligenten Volkes leitet sich auch die charakteristische Vorliebe für direkte Demokratie ab. Das populistische Volk wird im Sinne Rousseaus zum moi commun - einem Kollektivsubjekt mit homogenem Willen (vgl. Wildt 2017: 26). So folgt der Populismus zwar der demokratischen Idee der Volksherrschaft, steht aber gleichzeitig der repräsentativen Demokratie und letztlich auch dem Verfassungsstaat als Garant des Minderheitenschutzes skeptisch bis feindlich gegenüber (vgl. Decker 2013, S. 304). Populist:innen möchten , die vorhandene Interessenvielfalt in einer mehrheitsdemokratischen Entscheidungsbefugnis aufgehoben sehen“ (ebd.: 317). Dieses Demokratieverständnis macht eine homogene Volkskonstruktion notwendig und delegitimiert den bisweilen undurchsichtigen und daher ungeliebten Prozess der Kompromissaushandlung, an dessen Stelle die Dezision gesetzt wird, was den Populismus zu einer grundsätzlich antipluralistischen Ideologie macht (vgl. ebd.: 317f; Decker 2018, S. 20).

Neben die Unterscheidung von „Wir-da-unten“ und „Die-da-oben“ tritt im Rechtspopulismus ein „Wir“ und „die Anderen“. Die rechtspopulistische „Demokratie“" zieht ihre Legitimation aus der kulturellen Homogenität ihrer Bevölkerung, weshalb ein drohender Verlust dieser imaginären Gemeinschaft und ihres geteilten Wertesystems die nationale Einheit bedrohen würde (vgl. Corduwener 2014, S. 432). Rechts wird Populismus also, ,wenn die eigene Position identitätspolitisch durch eine ,kulturelle Abwertung der ,Anderen“ unterstrichen wird“ (Bebnowski 2015, S. 15). Im Kontext der europäischen Rechtspopulist:innen verengt sich dieses Othering zunehmend auf vermeintlich nicht-europäische Migrant:innen, die wiederum häufig als Muslim:innen markiert werden (vgl. Decker 2006, S. 17). Sie werden aufgrund ihres zugeschriebenen kulturellen Hintergrunds vom heartland ausgeschlossen, unabhängig von ihrer Staatsbürgerschaft (vgl. Betz 2005, S. 36), denn ,those who did not belong to the people of the heartland of the past do not belong to ,the people" of today, either" (Rydgren 2004, S. 206f). Die Basis, auf die sich diese Identität gründet, bleibt dabei häufig vage, was jedoch keineswegs zufällig ist. Einerseits erleichtert dies den Ausschluss verschiedener Outgroups, andererseits begünstigt ein lockerer Referenzrahmen das Märchen der Homogenität. Als gemeinsame Basis des heartlands wird somit immer häufiger das ,christliche Abendland“ und, im Falle der AfD, die deutsche Leitkultur herangezogen. „Dabei entsteht eine paradoxe Situation: Eine weitgehend sich säkular verstehende Gesell- 
schaft, die in religiösen Fragen indifferent ist, benennt als Kriterium der Differenz und Ausgrenzung das Religiöse“ (Kampling 2012, S. 163). Ursula Birsl (2017, S. 126) sieht in der sakralen Konstitution von In- und Outgroups den Vorteil der Variabilität sowie die inhärente Vorstellung von Ungleichwertigkeit und Hierarchie. Die Ideologie ,kann transnational, national oder ethnozentrisch ausgerichtet sein, ermöglicht jedoch im Grundsatz auch den Beitritt zur in-group unabhängig von nationaler oder ethnischer Zuschreibung“ (ebd.).

Populistische Parteien versuchen den vermeintlichen Konflikt zwischen Ingroup und Outgroup in bestehende gesellschaftliche Probleme einzubetten und werden „umso erfolgreicher sein, je mehr es ihnen gelingt, aus den ökonomischen, kulturellen und politischen Krisenerscheinungen gleichzeitig Kapital zu schlagen und sie zu einer programmatischen Gewinnerformel zu verbinden“ (Decker 2006, S. 15). Insofern ist es auch kein Zufall, dass sich die inhaltliche Verengung der AfD auf die Problematisierung muslimischer Zuwanderung mit den zunehmenden Fluchtbewegungen ab 2015 deutlich verstärkt hat. Man greift bewusst die Sorgen politisch orientierungslos gewordener Menschen auf und bettet sie in rechtspopulistische Konzepte ein, dabei geht es beispielsweise um die ,Verteidigung und Rückeroberung von Lebens- und Wohlstandsniveaus gegen die Bedrohung durch Immigration und um die Bewahrung nationaler und kultureller Identität" (Schönfelder 2013: 109). Die Ausrufung eines dauerhaften Krisenzustandes und die Umdeutung jeglicher Konflikte in einen Kampf der Kulturen sind daher ein taktisches Grundinteresse der Rechtspopulist:innen. Soziale Missstände würden indes ,,in ethnischen, kulturellen oder geschlechtlichen Differenzen vermutet“ (Salzborn 2018: 27).

Rechtspopulismus kann sowohl in demokratischer als auch in extremer Form auftreten (vgl. Thieme 2019, S. 6; Bauer 2014: 9), je nachdem, wie das heartland interpretiert wird. Die AfD bewegt sich dabei zunehmend an den rechten Rand und beweist, dass es gerade rechtsextreme Positionen sind, die unter dem Deckmantel von Kulturprotektionismus sowie Meinungs- und Religionsfreiheit geäußert werden und sich als besonders anschlussfähige Erfolgsformeln erweisen, wie etwa die steigende Zustimmung zu muslimfeindlichen Aussagen und die allgemeine Zunahme rechtsextremer Einstellungen innerhalb ihrer Wähler*innenschaft zeigt (vgl. Pesthy et al. 2020). Ein Grund für die Partei, derartige Themen bewusst auf die politische Agenda zu setzen. Dies spiegelt sich auch in der Wähler:innenschaft wider, die sich zunehmend rechts oder sogar weit rechts verortet (vgl. Celik et al. 2020, S. 164-168) und im Vergleich zur Gründungsphase ,rechtsextremer geworden ist“ (ebd.: 167). Gideon Botsch (2020) stellt daher fest, die AfD sei ,noch nicht rechtsextrem, muss aber nach wie vor als rechtsextrem dominiert betrachtet werden“. Sie artikuliert ihr rechtspopulistisches Weltbild mit Hilfe einer Volkskonstruktion, die dem klassischen Rechtsextremismus entlehnt ist. Die Instrumentalisierung und Verbreitung rechtsextremer Ansichten, insbesondere im Hinblick auf eine religionsbasierte Semantik, erweist sich dabei als gesellschaftlich anschlussfähig. Warum dies so ist, soll im folgenden Abschnitt dargelegt werden, ehe anhand einer Analyse von Programmen, Reden, Pressemitteilungen und Aussagen einzelner Funktionär:innen der empirische Nachweis für die Instrumentalisierung religiöser Bezugnahme erbracht wird. 


\section{Muslimfeindliche Kontinuitäten: der Anschluss der AfD an den deutschen Migrationsdiskurs}

Es zeigt sich, dass die AfD Minderheiten als ,fremde“ Outgroups konstruiert, einer ethnischen und kulturell homogenen Ingroup gegenüberstellt und somit kategorisch aus dem heartland ausschließt. Dabei greift sie in einer für (rechts-)populistische Bewegungen kennzeichnenden Art gesellschaftliche Krisen auf, stilisiert sie zu bedrohlichen Untergangsszenarien sowie konstruiert und benennt in simplifizierender Weise (Feind-)Gruppen, die - meist in kollaborierender Weise - als Verursacher:innen der Krisen ausgemacht werden. Die Wahlerfolge der AfD seit ihrer Gründung 2013 müssen somit als Produkt und Multiplikator einer Gesellschaft verstanden werden, in der in Teilen bereits eine Akzeptanz pluralitätsabwehrender und muslimfeindlicher Ansichten eines kulturell homogenen Nationalstaates sowie rechtsextreme, rassistische Einstellungen vorhanden waren (vgl. Yalcin 2019, S. 311). So stellte die AfD Stand Juli 2021 in drei Landtagen die zweitmeisten Abgeordneten und erhielt bei den Bundestagswahlen 2017 die drittmeisten Stimmen aller Parteien.

Diese Abwehr von Pluralität zeigt sich im Migrationsdiskurs der Bundesrepublik (und der DDR) bereits seit der Anwerbung der „Gastarbeiter“ in den 1950er Jahren, wobei an alte Muster des Umgangs mit ,Wanderarbeitern“ und „Fremdarbeitern“ zu Zeiten des Kaiserreichs angeknüpft wurde. Dass „Gastarbeiter“ eines Tages Teil der Gesellschaft sein sollten, war von vornherein nicht vorgesehen. Ein Anspruch auf rechtliche Gleichbehandlung über an die Erwerbstätigkeit gekoppelte sozialversicherungsrechtliche Leistungen hinaus oder gar eine Einbürgerung wurde a priori durch das „Ausländergesetz“ und die Staatsangehörigkeit nach dem Abstammungsprinzip (ius sanguinis) ausgeschlossen (vgl. Morgenstern 2002, S. 234ff.). Die Bundesrepublik sei kein Einwanderungsland, so der Tenor, da sie seit jeher zu dicht bevölkert gewesen sei (vgl. ebd.: 252). Die Religion und Kultur der „Gastarbeiter“ fand im politischen Diskurs keine große Aufmerksamkeit (vgl. Attia 2015, S. 183ff.). Im Rahmen der Diskussion um „Ghetto-Bildungen“ in bundesdeutschen Ballungszentren wurde Ende der 1970er-Jahre fortschreitend der potenziell längerfristige Aufenthalt der „Gäste“ erkannt, weshalb sich im parlamentarischen Diskurs eine Verschiebung der Bezeichnung „Gastarbeiter“ hin zum „Ausländer“ vollzog (vgl. Morgenstern 2002, S. 252). Die „Ausländer“ gerieten in der Folge besonders aufgrund der wachsenden Anzahl der „Ausländerkinder“ in den Mittelpunkt der Debatten. Sie fielen durch „Integrationsprobleme“ auf, die auf ihre Abstammung zurückgeführt wurden (vgl. ebd.: 259). Ein besonders problematisches „Integrationsdefizit“ wurde bei nicht-europäischen Zuwander:innen ausgemacht, deren unterschiedliche Kultur ein generelles Problem darstelle. So schaffte es der Begriff „Kultur“ im Lauf der 1980erJahre als ,,zeitgemäßes, gleichermaßen naturalisierendes wie mystifizierendes“ (ebd.: 343) Differenzkonzept in den öffentlichen Diskurs. Probleme der „Ausländer“ wurden somit vermehrt in „Kulturfremden“ gesucht, der „Türke“ wurde zum Symbol von Gewaltkriminalität, Belastung der Sozialsysteme und somit gescheiterter Integration (vgl. ebd. 345; Çağlar 1990, S. 9). Die Dichotomie zwischen den „Deutschen“ und den „Ausländern“ dominierte somit in verschiedenen Phasen und Ausprägungen den bundesdeutschen Diskurs, immer unter der Prämisse, dass die Bundesrepublik kein Einwanderungsland sei. Eine Änderung brachte 1998 der Regierungswechsel hin 
zu einer rot-grünen Koalition. In der Koalitionsvereinbarung formulierten SPD und Grüne den Satz: ,Wir erkennen an, dass ein unumkehrbarer Zuwanderungsprozess in der Vergangenheit stattgefunden hat“ (Bündnis 90/Die Grünen/SPD 1998, S. IX) und gaben somit die Behauptung vom „Nichteinwanderungsland“ Deutschland auf. Damit einher ging die Reform des deutschen Staatsangehörigkeitsgesetzes, das in der Folge die doppelte Staatsangehörigkeit in bestimmten Fällen ermöglichte und eine Abkehr vom Abstammungsprinzip (ius sanguinis) zum Territorialprinzip (ius soli) festlegte.

Durch die Öffnung des Staatsangehörigkeitsrechts wurde die nationale Zugehörigkeit als Differenzkategorie in ihrer Wirksamkeit beschnitten. Die „Imagination von Reinheit und Exklusivität“" (Foroutan 2019, S. 38) geriet ins Wanken, und etablierte ethno-nationale Klassifizierungsmechanismen wurden in Frage gestellt. Dies hatte den Beginn einer Neubestimmung des nationalen Selbstverständnisses zur Folge, in dessen Fokus der „muslimisierte Migrant“ (vgl. Karakaşoğlu 2009, S. 186) als neue Fremdkategorie rückte (vgl. Spielhaus 2013, S. 171). Während der muslimische Glaube der „Gastarbeiter“ nur selten thematisiert worden war (vgl. Attia 2015, S. 185), ließ sich nun eine „Islamisierung der Debatten“ (Tiesler 2006) beobachten. Verbunden wurde dies mit einer Bezugnahme auf die Differenzlinie Kultur, die sich ganz im Duktus von Huntingtons (1996) „Kampf der Kulturen“ entlang religiöser Markierungen erstreckte. Debatten um eine deutsche „Leitkultur“ unterstrichen die neuen Differenzlinien (vgl. Pautz 2005). Muslim:innen galten fortan als per se schwieriger zu integrieren als andere Migrant:innen, und Integrationsprobleme sowie sozioökonomische Defizite, die bislang mit Ausländer:innen oder sozialen Schichten im Allgemeinen in Verbindung gebracht wurden, wurden nun vornehmlich mit Muslim:innen assoziiert (vgl. Hierl 2012, S. 59). Auf politischer Ebene widmete sich der deutsche Staat explizit dem Islam, indem 2006 die Deutsche Islam Konferenz (DIK) als Forum für eine ,notwendige“ Integration von Muslim:innen in der Bundesrepublik durch das damalige Innenministerium ins Leben gerufen wurde. Muslim:innen wurden somit als besonders nennenswert ,zu integrierende“ Bevölkerungsgruppe hervorgehoben und zum „Anderen“ stilisiert (vgl. Tezcan 2012; AmirMoazami 2009). Dieses skeptische bis ablehnende Stimmungsbild spiegelte sich auch in der Bevölkerung wider, wie Studien zur „Gruppenbezogenen Menschenfeindlichkeit“ zeigen: 2003 stimmte rund ein Drittel der Befragten der Aussage „Durch die vielen Muslime fühle ich mich manchmal wie ein Fremder im eigenen Land“ zu (vgl. Leibold und Kühnel 2003, S. 103).

Ihre öffentliche Artikulation fanden explizit muslimfeindliche Thesen im 2010 erschienenen Pamphlet des damaligen SPD-Politikers Thilo Sarrazin ,Deutschland schafft sich ab“. Das Buch entpuppte sich als Kampfschrift gegen „muslimische Zuwanderung“. Die „Fruchtbarkeit“ von Muslim:innen stelle auf lange Sicht eine Bedrohung für das alternde Europa dar, da ihre Wertvorstellungen einen kulturellen und zivilisatorischen Rückschritt bedeuten, so Sarrazin (vgl. Sarrazin 2010, S. 267). Der Autor hatte bereits im Vorfeld der Veröffentlichung in einem Interview mit der Zeitschrift Lettre International rassistische Äußerungen gegenüber Muslim:innen von sich gegeben, als er konstatierte, er müsse ,niemanden anerkennen, der vom Staat lebt, diesen Staat ablehnt, für die Ausbildung seiner Kinder nicht vernünftig sorgt und ständig neue kleine Kopftuchmädchen produziert“" (Berberich und Sarra- 
zin 2009, S. 199). Sein Werk verkaufte sich innerhalb weniger Monate über eine Million Mal und wurde zum meistverkauften Sachbuch in Deutschland (vgl. Hafez 2019, S. 96). Viele seiner Thesen erfuhren aufgrund der rassistischen Wortwahl zwar öffentlich Verurteilung, im Kern teilten aber nicht wenige Kommentator:innen seine Aussagen, da er bestehende Probleme ansprechen würde (vgl. Cremer 2019, S. 113). Dies spiegelte sich auch in damaligen Umfragen, die einer fiktiven Sarrazin-Partei bis zu $18 \%$ der Stimmen prognostizierten (vgl. Häusler und Roeser 2014, S. 29f.). Im Zuge des Anstiegs der Geflüchtetenzahlen 2015 etablierte sich die bereits weitestgehend synonyme Nutzung der Kategorien „Migrant“" und „Muslim“ (vgl. Spielhaus 2018, S. 135).

Es zeigt sich, dass migrationsabwehrende Diskurse in Deutschland bereits vor dem Eintritt der AfD in das politische Feld existierten und in der Ablehnung von Muslim:innen Ausdruck finden. Wie sich den Studien zur „Gruppenbezogenen Menschenfeindlichkeit" entnehmen lässt, ist die Zustimmung zu islamfeindlichen Statements zwischen 2001 und 2011 stetig gestiegen, mit einer signifikanten Spitze im Jahr 2010, dem Jahr, in dem Sarrazins Buch erschien (vgl. IKG2011). Auch die jüngste Leipziger Autoritarismus Studie (LAS) bestätigt nach wie vor hohe Zustimmungswerte zu muslimfeindlichen Aussagen (vgl. Decker et al. 2020, S. 64). Einwanderungs- und Terrorismusdebatten erzeugen soziale und identitäre Spannungen, in deren Kontext bis heute Fragen um nationale Zugehörigkeit und die Rolle von Migration - im Speziellen die Rolle der Muslim:innen und des Islams - verhandelt werden (vgl. Foroutan 2019, S. 17). Migration hat sich dadurch zum „Meta-Narrativ“ (ebd.: 154) entwickelt, über das Grundfragen des gesellschaftlichen Zusammenlebens thematisiert werden. Migration und die Rolle von Muslim:innen kristallisiert sich als Chiffre für gesellschaftliche Pluralität heraus, und ihre Abwehr kann als Angst vor Privilegienverlust verstanden werden (vgl. ebd.: 14; 118).

Wenn zunehmend mehr Menschen für sich in Anspruch nehmen deutsch zu sein [...], dann erzeugt diese Hybridisierung der Zugehörigkeit [...] Ambivalenz. Kulturelle und symbolische Begründungen werden herangezogen, um dieser migrantisch markierten Gruppe das Versprechen der Demokratie vorzuenthalten (ebd.: 23).

Statistische Erhebungen zur Outgroup Mobility Threat zeigen deutlich, dass seitens der Mehrheitsgesellschaft eine Bedrohung im Aufstieg der „Anderen“ ausgemacht wird (vgl. Foroutan et al. 2019). Auch die LAS (vgl. Pickel et al. 2020, S. 105) belegt einen signifikanten Zusammenhang zwischen der fehlenden Anerkennung von Pluralität und Toleranz und antimuslimischen Haltungen, gepaart mit einem ausgeprägten Nativismus. Der per se nativistisch argumentierende Rechtspopulismus greift diese muslim- und islamfeindlichen Haltungen auf und verknüpft sie mit Identitätsfragen, um potenziellen Wähler:innen scheinbare Antworten auf Globalisierungs- und Überfremdungsängste zu liefern. Wie die AfD Pluralisierungsängste und Muslimfeindlichkeit für sich nutzt, soll in der Folge anhand einer empirischen Analyse der Parteiprogramme und Aussagen von Parteifunktionär:innen verdeutlicht werden. 


\section{Antimuslimischer Populismus: die AfD und der Islam}

Vor dem Hintergrund antimuslimischer Ressentiments im bundesdeutschen Diskurs bieten der AfD vorrangig Muslim:innen ein wirkmächtiges Angriffsziel (vgl. Häusler 2019, S. 8). 2013 als Anti-Euro-Partei in Folge der Finanzkrise gegründet war das oberste Ziel zunächst jedoch die nationale Emanzipation gegenüber der EU und die Abschaffung des Euros. Dennoch ließ Parteigründer Bernd Lucke in seinem Thesenpapier zum Islam bereits erkennen, dass eine implizite Bejahung des Satzes „Der Islam gehört zu Deutschland“ „falsch und töricht“ (vgl. Lucke 2013), da undifferenziert, sei. Die neu gegründete AfD vereinigte neben Wirtschaftsliberalen vor allem nationalkonservative, neurechte sowie rechtspopulistische Kräfte (vgl. Häusler 2017, S. 69; Häusler und Roeser 2015, S. 28-41), und im Zuge des Aufkommens der Bewegung Pegida erweiterte sie ihre politische Agenda um das Thema Einwanderung und die daran gekoppelte Mobilisierung gegen den Islam (vgl. Korsch 2016). Im Juli 2015 zog sich Bernd Lucke nach der Niederlage bei der Wahl zum AfDBundessprecher gegen Frauke Petry aus der Partei zurück, und der parteipolitische Kurs verschärfte sich hin zu einem ,Rechtspopulismus mit völkisch-nationalistischen Merkmalsprägungen“ (Häusler 2017, S. 69). Die AfD griff somit endgültig die muslimfeindlichen Einstellungen auf, die in Pegida ein ,niedrigschwelliges Angebot zur Artikulation auf der Straße“ (ebd.: 66) erhalten hatten. Die seit Jahrzehnten in der Bundesrepublik verankerten nationalistischen, migrationsabwehrenden und muslimfeindlichen Einstellungen erfuhren dadurch auf realpolitischer Handlungsebene eine wirkmächtige parteipolitische Entsprechung (vgl. ebd.: 71).

So konstatiert die Partei wenig später in ihrem Grundsatzprogramm „Der Islam gehört nicht zu Deutschland“ (AfD 2016, S. 49) und lehnt ,die Ideologie des Multikulturalismus“ zugunsten einer „,deutschen Leitkultur“ (ebd.: 47) ab. Darüber hinaus betont sie - im Rückgriff auf bundesdeutsche Argumentationen aus den 1980er-Jahren -, dass es sich bei Deutschland ,aufgrund seiner geografischen Lage, seiner Geschichte, Bevölkerung und dichten Besiedelung“ (ebd.: 58) um kein Einwanderungsland handle. Analog dazu fordert die AfD eine Rückkehr zum Staatsangehörigkeitsgesetz nach dem Abstammungsprinzip, wie es bis zum Jahr 2000 galt (vgl. AfD 2017, S. 32). Begründet wird dies damit, dass die deutsche Staatsbürgerschaft untrennbar mit ,unserer Kultur und Sprache“ verbunden sei und nur an mündige Einwanderer vergeben werden sollte (vgl. AfD 2016, S. 65). Hier wird das Verständnis einer an die Abstammung gekoppelten quasi-natürlichen Kultur deutlich. Die AfD greift somit sowohl den antimuslimischen als auch den Antimigrationsdiskurs auf, der noch bei der Gründung der Partei keine tragende Rolle gespielt hatte.

In ihrer politischen Agitation gegen Pluralisierung und Multikulturalismus hebt die AfD das etablierte „Feindbild Islam“ (Hafez 2019) hervor und gibt dabei unter dem Deckmantel von „Islamkritik“ und Kulturprotektionismus muslimfeindliche Äußerungen von sich, die als antimuslimischer Rassismus (vgl. Attia 2009; Shooman 2014; Häusler 2019) eingestuft werden müssen und rechtsextremen Charakter aufweisen. In diesen Äußerungen steht nicht das muslimische Individuum im Vordergrund, sondern eine Gemeinschaftsidentität (vgl. Hafez 2019, S. 15), die als „,fremd“ dargestellt und in einen unveränderlichen Gegensatz zum homogenen „deutschen“ Volk gesetzt wird. Antimuslimische Agitation dient damit gleichsam als Abwehr 
demokratischer Pluralisierung und als Mittel der völkisch-nationalen Identitätspolitik. Dies erfolgt im besonderen Maße über den Marker „Kultur“. So betrachtet die AfD die ,geschichtsblinde“ Gleichstellung der importierten „kulturellen“ Strömungen mit der „,einheimischen Kultur“ „als ernste Bedrohung“ (AfD 2016, S. 47), und ,der in Europa bereits stattfindende Kulturkampf zwischen Abendland und dem Islam“ (AfD 2017, S. 47) könne nur durch Abwehrmaßnahmen verhindert werden. Dabei bemüht die Partei das Bild des aufgeklärten „Abendlandes“, das im Widerspruch zur mystifizierten, irrationalen Heilslehre des Islam stehe (vgl. ebd.). Der Antagonismus zwischen einem emanzipativen, demokratischen, fortschrittlichen und zivilisierten Westen mit „christlich-abendländischem“ Erbe einerseits und dem rückständigen, unwandelbaren und unaufgeklärten Islam (vgl. Shooman 2014, S. 62) andererseits kulminiert im Bild der Muslim:innen als unveränderliche, quasinatürliche Träger:innen ihrer Kultur. Es erfolgt eine rassistisch unterfütterte Generalisierung ,Muslim = Ausländer + Islamist + Kulturzerstörer + Eroberer“ (Häusler 2019, S. 10). Somit werden u.a. auch deutsche Muslim:innen zu „Fremden“ erklärt. Legitimiert wird dies durch eine Ethnisierung und Kulturalisierung sozialer und gesellschaftlicher Konflikte, die ihre Hypostasierung in der Figur des „Muslims“ findet. Eine (einseitig verstandene) Integration wird dabei als unmöglich dargestellt, da sie nur im Falle einer Assimilation erfolgen könne, die aber nicht erzwingbar sei (vgl. AfD BB 2019, S. 57). Mit Verweis auf verschiedene „Mentalitäten“ (AfD BW 2016, S. 25) werden kulturelle Differenzen essenzialisiert und Integration als „,von Natur aus" unmöglich dargestellt - und dabei gilt: Wer sich nicht integrieren könne, der ,wird [hier] keine Heimat finden“ (AfD TH 2019, S. 50).

Die Religion spielt somit im Kern nicht die primäre Rolle in der antimuslimischen Mobilisierung der AfD. Sie wird für kulturalisierende Zwecke missbraucht, um eine Outgroup festzulegen. Gleichzeitig wird Religion als diskursives Element mit politischen Paradigmen vermischt, um Machtansprüche der „ethno-natio-kulturellen“ (Karakayali und Mecheril 2018, S. 230) Ingroup unter dem Deckmantel eines modernen Säkularismus zu manifestieren (vgl. Keskinkılıç 2019, S. 47). Grundsätzlich sieht die AfD den Islam ,im Spannungsverhältnis mit unserer Werteordnung“ (AfD 2016, S. 48), die sie als „freiheitlich-demokratisch“ bezeichnet. Ein Islam, der ,unsere" Rechtsordnung nicht respektiere und einen Herrschaftsanspruch erhebe, sei mit „unserer“ Kultur nicht vereinbar. Neben kulturalistisch-religiösen Argumenten einer Inkompatibilität treten somit unvereinbare politische Ansprüche ,des Islam“ zu Tage. Diese werden anhand von Religionspraktiken symbolisch festgemacht: So seien das Minarett und der Muezzin-Ruf islamische Herrschaftszeichen und ,religiöser Imperialismus“ (AfD 2017, S. 35). Das Tragen eines Kopftuchs stelle ein religiöspolitisches Zeichen dar (AfD 2016, S. 50). Die AfD Sachsen lehnt den islamischen Religionsunterricht an Schulen mit Verweis auf „,den politischen Islam“ als „,totalitäre Ideologie, die unserer Kultur feindlich gegenüber steht und mit dem Grundgesetz nicht vereinbar ist" (AfD SN 2019, S. 34), ab. Eine direkte Verbindung von Muslim:innen und Islam in Deutschland mit islamisch-theokratischen Staaten stellt die AfD Brandenburg her, wenn sie konstatiert, die Todesstrafe, Unterdrückung von Frauen und Mädchen oder fehlende Toleranz gegenüber anderen Weltanschauungen, „wie sie in islamischen Ländern an der Tagesordnung sind“ (AfD BB 2019, S. 56), hätten im aufgeklärten Brandenburg nichts zu suchen. In der von der AfD-Fraktion 
im Thüringer Landtag herausgegebenen Publikation „Der Islam. Fakten und Argumente“ (AfD-thl 2017, S. 34) bringt die AfD ihre Sichtweise auf den Punkt: „,Der Islam“ sei eine ,politische Religion“. Den ausgemachten politischen Herrschaftsanspruch des Islam nimmt die Partei folglich zum Anlass, Restriktionen für die Religionsausübung von Muslim:innen einzufordern. Postuliert wird dies im höchst widersprüchlichen Satz: „Die AfD bekennt sich uneingeschränkt zur Glaubens-, Gewissens- und Bekenntnisfreiheit. Sie fordert jedoch, der Religionsausübung durch die staatlichen Gesetze, die Menschenrechte und unsere Werte Schranken zu setzen“ (AfD 2016, S. 95). Diese Aussage ist in mehrerlei Hinsicht problematisch: Einerseits erhebt sich die Religionsfreiheit im Artikel 4 des Grundgesetzes nicht über die Menschenrechte. Andererseits ist sie selbst Menschenrecht, darf aber in der Bundesrepublik nicht zur Beschneidung anderer Menschenrechte instrumentalisiert werden. Der Ausspruch, Glaubens- und Bekenntnisfreiheit sei erwünscht, Religionsfreiheit müsse aber beschnitten werden, steht offenkundig im Widerspruch zueinander, Artikel 4 im Grundgesetz trennt „Religion“ nicht von „Glaube“ (vgl. Art 4 GG). Zudem diskreditiert die AfD dieses Grundrecht, indem sie es überhöhend als ein „Supergrundrecht“ (AfD TH 2019, S. 53) diffamiert, durch das sich muslimische Gemeinschaften einen politischen und gesellschaftlichen Sonderstatus sicherten (vgl. ebd.). Daher gelte es für die AfD, dieses Grundrecht entgegen allen vorangegangenen Bekenntnissen zu beschneiden: So möchte sie neben den Ausrufen des Muezzins das Tragen von Kopftüchern im öffentlichen Dienst und Bildungseinrichtungen verbieten (vgl. AfD 2016, S. 50), öffentliche muslimische Freitagsgebete untersagen (vgl. AfD BY 2018, S. 20) und keine weiteren Moscheebauten, besonders mit Minarett, genehmigen (vgl. AfD SN 2019, S. 22) - die freie Religionsausübung für Muslim:innen sei bereits gewährleistet und Moscheen für eine freie Religionsausübung nicht notwendig (vgl. ebd.: 22, 44). In ihren Forderungen nach Einschränkungen der Religionsfreiheit von Muslim:innen stilisiert die AfD den Islam durch eine Gleichzeitigkeit von „Nicht-Religion“ (politische Ideologie) und „Zu-viel-Religion“ (Religionspraxis muss beschnitten werden) zu einer Bedrohung für die säkulare Ordnung (vgl. Keskinkılıç 2019, S. 48).

Fragen der Religionsausübung und -freiheit dienen aber primär als Vorwand für die antimuslimische Abwehrhaltung. Im Kern begründet die Partei den nativistisch motivierten Ausschluss der Muslim:innen aufgrund einer anders gearteten Kultur mittels rassifizierender Techniken der diskursiven Zuschreibung (vgl. Bühl 2017, S. 133). Dabei greift sie auf ein breites Wissensnetz orientalistischer Diskurse zurück, wie dies in antimuslimischer Rhetorik häufig der Fall ist (vgl. Attia 2009; Shooman 2014; Keskinkılıç 2019). Vertreter:innen der Partei bedienen sich in ihren Äußerungen zum Islam eines bereits etablierten antimuslimisch-rassistischen Duktus. So wird dogmatisch eine Demographisierung (vgl. Bühl 2017, S. 177) vorgenommen, die Muslim:innen eine hohe Geburtenrate mit der Absicht einer Unterwanderung und „Islamisierung“ der Mehrheitsgesellschaft bescheinigt. Dies wird regelmäßig mit parasitären Zuschreibungen verbunden. Die Nutzung etablierter Phrasen in Anlehnung an Sarrazin zeigen sich etwa in der Forderung ,Deutschland nicht abschaffen" (AfD 2017, S. 37) oder in der öffentlichkeitswirksamen Ansage der AfDCo-Vorsitzenden im Bundestag Alice Weidel, dass „Burkas, Kopftuchmädchen und alimentierte Messermänner“ (Weidel 2018) den Sozialstaat nicht voranbringen wür- 
den. Das Bedrohungsszenario einer „Islamisierung“ lässt sich in mehreren Landtagswahlprogrammen der AfD wiederfinden. Die AfD Bayern wendet sich gegen eine „Islamisierung des öffentlichen Raums“ (AfD BY 2018, S. 47) und die AfD Sachsen gegen eine „,schleichende Etablierung islamischer Strukturen“(AfD SN 2016, S. 44). Die AfD Brandenburg verweist auf die negativen Entwicklungen, die mit der Einwanderung aus dem ,orientalischen Raum“ (AfD BB 2019, S. 58) einhergingen. Die AfD Niedersachsen prognostiziert gar eine ,Verdrängung der einheimischen deutschen Bevölkerung“ (AfD NI 2017, S. 77) bis zum Ende des Jahrhunderts. Deutlich wird die Stilisierung von Expansions- und Unterwanderungsszenarien auch in Statements, die Ähnlichkeiten zu rechtsextremen Verschwörungstheorien eines „Großen Austauschs“" aufweisen (vgl. Häusler 2019, S. 37). So verweist der AfD-Bundestagsabgeordnete Gottfried Curio auf den „Geburten-Jihad“ (Curio 2018), durch den in Kürze Millionen „Ausländer“ „unser“ politisches Schicksal mitbestimmten. Der Fraktionsvorsitzende der AfD im Thüringer Landtag Björn Höcke bezeichnet den Islam als „Besatzungsmacht“ (zit. n. Häusler 2019, S. 41) und beschwört „rassistische Säuberungsphantasien“ (Häusler 2019, S. 41), wenn er von einem ,großangelegten Remigrationsprojekt“ (ebd.) spricht. In einer Publikation der Thüringer AfD zum Islam werden darüber hinaus Expansionsabsichten als dem „,militaristischen“ Islam wesensimmanente Gewaltaspekte hervorgehoben (vgl. AfD-thl 2017, S. 92f.). All diesen Äußerungen lässt sich entnehmen, dass die ,Islamisierung“ laut der AfD vor allem mit der „Waffe“ Geburt operiere. Dagegen müsse daher ein ,deutscher Geburtenkampf“"geführt werden (vgl. Keskinkılıç 2019, S. 77). Dies zeigt sich an einem Wahlkampfplakat der AfD mit der Abbildung einer weißen schwangeren Frau und der Betitelung „,Neue Deutsche?‘ Machen wir selber!“(vgl. ebd.). Die AfD knüpft somit an die „Sorgen“ der Bürger:innen vor einer „Islamisierung“ an, die bereits in der muslimfeindlichen Mobilisierung der Pegida-Bewegung, aber auch in der Befürchtung einer steigenden Anzahl „nicht integrierter Ausländerkinder“ in den 1970er- und 80er-Jahren zum Ausdruck kamen.

Neben rassistischen Überfremdungs- und Unterwanderungsszenarien bemüht die AfD die Figur des homophoben und frauenfeindlichen „Muslims“, die gleichzeitig zur Inszenierung der eigenen „Offenheit“ instrumentalisiert wird und somit widersprüchlich zur antipluralistischen Politik der Partei ist. Das Tragen eines Kopftuchs lehnt die AfD generell ab, da es der Gleichberechtigung widerspreche und die „Unterordnung von muslimischen Frauen unter den Mann“ (AfD 2016, S. 99) symbolisiere. Neben der Unterdrückung werde auch eine Form der Sexualisierung vorgenommen, die ein ,freies und selbstbestimmtes Leben behindert“ (AfD BB 2019, S. 38). Indem sie ein selbstbestimmtes Tragen des Kopftuchs ausschließt, stellt die AfD Musliminnen somit a priori als unmündig dar und entindividualisiert sie gleichsam. Weitere Gleichberechtigungsthemen, die einer legitimen Kritik unterzogen werden könnten, werden mit rassistisch stilisierten Verweisen auf „,Miniharems“, ,polygame Verhältnisse“ sowie eine restriktive und bigotte Sexualmoral islamischer Gesellschaften verklausuliert, die eine generalisierende und depersonalisierende Wirkung haben sowie Frauenfeindlichkeit als ein genuines Problem des Islam und somit der „Fremden“ darstellt (vgl. Keskinkılıç 2019, S. 49f.). 2017 nutzte Alice Weidel ihr offizielles Coming-Out in der Partei, um Homophobie- und Sexismus-Vorwürfe von der AfD zu weisen und gleichzeitig auf die „Gefahr“ der 
Muslim:innen hinzudeuten (vgl. ebd.: 52). Sie sei nicht trotz ihrer Homosexualität, sondern gerade deswegen Mitglied der AfD, denn „muslimische Gangs machen in letzter Zeit förmlich Jagd auf Homosexuelle und das mitten in Deutschland“ (Weidel 2017). Die Berliner AfD wirbt auf einem Landtagswahlplakat mit dem Bild zweier Männer und der Überschrift „,Mein Partner und ich legen keinen Wert auf die Bekanntschaft mit muslimischen Einwanderern, für die unsere Liebe eine Todsünde ist" (vgl. Keskinkıliç 2019, S. 52, herv. i. O.). Das gesellschaftliche Thema der Homophobie wird von der AfD somit ebenfalls ausschließlich in der Arena der Islamdebatten verortet, um die „Anderen“ als Projektionsfläche für Probleme zu nutzen, die somit von „uns“ abgewiesen werden können. Misogynisierung und Homophobisierung (vgl. Bühl 2017, S. 186) können als sexualitätsbezogene rassistische Praktiken des Ausschlusses von Muslim:innen verstanden werden. Religion ist in dieser Argumentation nur der scheinbar primäre Anknüpfungspunkt und wird mit vermeintlich politischen Absichten sowie einer rückschrittlichen Kulturzuschreibung des Islam verbunden. Somit wird die Outgroup als Bedrohung für die Ingroup inszeniert, die Anerkennung von Pluralität versagt und als Begründung für Migrationsabwehr herangezogen.

\section{Christentum, Judentum, Islam: eine instrumentelle Dreiecksbeziehung}

Da im Rahmen des deutschen Staatsangehörigkeitsgesetzes „deutsch“ keine exklusiv ethnisch-national vererbte Kategorie mehr ist, sondern die Zugehörigkeit auch durch Einbürgerung erworben werden kann, sind ,deutsch“ und ,muslimisch“ längst einander überschneidende Kategorien geworden. Der Verweis auf das eigene „Deutsche“ genügt somit nicht mehr, um nativistische Positionen zu begründen. Daher instrumentalisiert die AfD etablierte Muster des orientalistischen Islamdiskurses, indem sie Europa als ,christliches Abendland“ stilisiert und den Islam in Said'scher Logik als kulturellen Gegenspieler konstruiert (vgl. Said 2017 [1978]). So kann im antipluralistischen Diskurs in Kombination mit der Essentialisierung der ,anderen“ Kultur gewährleistet werden, dass ein ethnisch heterogenes sowie multi- bzw. transkulturelles deutsches Staatsvolk durch den Eintritt muslimischer Menschen ins heartland trotz Staatsbürgerschaftserwerb unmöglich, da kulturell unvereinbar bleibt. Für die exklusive populistische Homogenitätskonstruktion erweist sich eine „ethnisierte Religionszugehörigkeit“" als hilfreiches Werkzeug (vgl. Pickel 2018, S. 309). Daher wird die AfD nicht müde, in ihren Aussagen auf das christliche Kulturerbe Deutschlands und Europas zu verweisen. Dies geschieht in verschiedenen Modi, die allesamt ein überhöhtes Bild des christlichen Europa dem einer rückständigen ,,islamischen Welt“ gegenüberstellen. So basiere die „deutsche Leitkultur“ laut der AfD im Wesentlichen auf drei Quellen: der religiösen Überlieferung des Christentums, der wissenschaftlich-humanistischen Tradition und dem römischen Recht (vgl. AfD 2016, S. 92). Erweitert wird dies durch die ,jüdisch-christlichen (...) Grundlagen unserer Kultur" (vgl. ebd.: 95), die durch die islamische Glaubenspraxis bedroht seien. Lokalisiert wird diese Kultur im „Abendland“ Europa, also außerhalb des Einflussbereiches des islamisch geprägten „Kulturkreises“. Dass sich im Topos des 
„Abendlandes“ der eigenen Kultur zugeschriebene Attributionen relativ lose kombinieren lassen, zeigt die AfD in ihrer diffusen Nutzung: So wird neben ,jüdischchristlichen“ Werten auf „christlich-abendländische“ (AfD HB 2019: 10; AfD BB 2019, S. 57), ,humanistisch-abendländisch[e]“ (AfD NRW 2017, S. 13) und ,,christliche und (...) aufklärerische“ (AfD BW 2016, S. 4) Werte bzw. Wurzeln verwiesen. Das „christliche Abendland“ hat sich somit zu dem Bezugsrahmen der Partei entwickelt, um eine Grenze zwischen In- und Outgroup zu ziehen. Auffällig ist dabei einerseits, dass die Rechtspopulist:innen ausgerechnet in der Säkularität, verstanden als die Trennung zwischen Kirche und Staat, ein spezifisch christliches Politikverständnis verorten, das dem politisierten Islam gegenübergestellt wird (vgl. Hidalgo et al. 2019, S. 3). Andererseits wird damit auch die Pluralität des Christentums verleugnet (analog zur jener des Islam), weshalb das Konzept der europäischen Heterogenität keineswegs gerecht wird (vgl. Hidalgo et al. 2019: 5). Diese Verwischung europäisch-christlicher Identitäten steht dabei auch im Widerspruch zu der ansonsten propagierten „Einheit in Vielfalt“ in einem „Europa der Vaterländer“. Dies fokussiert im Wesentlichen auf einen einzigen Punkt: Das „christliche Abendland“ ist nicht muslimisch. Dementsprechend muss der Verweis auf das Christentum als „matter of belonging rather than believing“ (Brubaker 2017, S. 1199) verstanden werden, da die AfD weder auf einen breiten christlichen Wähler:innenkreis noch auf eine besonders gute Beziehung zu den christlichen Kirchen blicken kann. Im Gegenteil: Eine tiefere Verbindung zur Kirche korreliert in Ostdeutschland mit einer niedrigeren Wahlwahrscheinlichkeit einer Zustimmung für die AfD (vgl. Huber und Yendell 2019, S. 76), und die Kirchen sprachen sich im Vorfeld der Bundestagswahl 2017 eindeutig gegen die Partei aus (vgl. Neuerer 2017). Negative Bezugnahmen sind indes durchaus beidseitig zu beobachten. Die AfD Bayern bezieht die Kirche in ihre populistische Elitenkritik mit ein, indem sie die Unterstützung der bayrischen Landesregierung für den ,kirchlichen Lobbyismus“ missbilligt (vgl. AfD BY 2018, S. 12). Dabei geht die Partei so weit, eine klare Trennung von Staat und Kirche zu fordern, und begründet dies unter anderem mit der hohen Anzahl an bekenntnislosen Bürger:innen, was als Widerspruch zu einer Politik für „,das christliche Volk“ im „Abendland“ gewertet werden kann. Die AfD Thüringen sieht die Kirche gar in einem Konglomerat mit den „Führungskadern der Altparteien“ und den Gewerkschaften, die den „Willen des Volkes“ missachten und die ,aggressive Anspruchshaltung bestimmter Zuwanderergruppen" (AfD TH 2019, S. 54) unterstützen würden.

Konkreter Ausgangspunkt für diese Ablehnung ist unter anderem die Tatsache, dass sich 2015 und auch 2016 viele Kirchenvertreter:innen für eine offene Aufnahmepolitik in der Flüchtlingskrise einsetzten, die auch der Vorsitzende der Deutschen Bischofskonferenz Kardinal Marx bestätigte (vgl. Weber 2017, S. 86). Das veranlasste die stellvertretende Bundessprecherin der AfD, Beatrix von Storch, dazu, die Abschaffung der Kirchensteuer anzuregen (vgl. Bax 2017, S. 83), und Rainer Rösl, Mitglied des „Pforzheimer Kreises“ (auch bekannt als Arbeitskreis „Christen in der AfD“ (ChrAfD)), äußerte, dass sich Christen lieber bei Pegida engagieren sollten (vgl. Kemper 2016, S. 11).

Sowohl die stetige Islamisierung politischer Debatten als auch die instrumentelle à-la-carte-Vereinnahmung des Christentums kann beispielhaft an der Familienund Bevölkerungspolitik der AfD veranschaulicht werden. Über die „Zivile Allianz“ 
(früher: „Zivile Koalition“) von Beatrix von Storch und ChrAfD finden christlichfundamentalistische Strömungen Anschluss an die AfD (vgl. Kemper 2020). Hier bildet die Partei mit ihrem Bekenntnis zur Privilegierung der heterosexuellen Mehrkindfamilie und ihrer Verteidigung klassischer Geschlechterrollen die Ansichten konservativer Christ:innen durchaus ab (vgl. Lang 2017: 63f.). Unter dem Stichwort „Willkommenskultur für Neu- und Ungeborene“ (AfD 2016, S. 44) setzt sie sich für ein weitreichendes Abtreibungsverbot ein und bedient sich dabei nicht zufällig einer Semantik der „Flüchtlingskrise“. Die AfD liegt hier ganz auf einer Linie mit fundamentalistischen Akteur:innen, wie den „Lebensschutz"-Bewegungen, die in Schwangerschaftsabbrüchen nicht nur einen zutiefst unchristlichen Vorgang sehen, sondern auch den Verfall des ,christlichen Abendlandes“, das am demographischen Wandel und Abtreibungen zugrunde gehe (vgl. Jentsch 2016, S. 100f.). Somit entpuppt sich die Ablehnung der Abtreibung innerhalb der AfD auch und vor allem als Kampf gegen eine vermeintliche Überfremdung (vgl. ebd.: 104). Sie fordert „,[m]ehr Kinder statt Masseneinwanderung“ (AfD 2016, S. 41), beklagt, „,[d]ass die Geburtenrate unter Migranten [...] deutlich höher liegt als unter deutschstämmigen Frauen“ (ebd.: 42), wodurch es einen ,ethnisch-kulturellen Wandel der Bevölkerungsstruktur" (ebd.) gebe, und suggeriert mit einem Verweis auf das vermeintlich geringere Bildungsniveau muslimischer Migrant:innen, dass es dadurch zusätzlich zu einer Verdummung des Landes kommen würde (vgl. ebd.). Letztlich erinnert die selektive Logik der Bevölkerungspolitik unweigerlich an die menschenverachtende Eugenik im Dritten Reich (vgl. Baier 2019) und liegt damit im Widerspruch zum christlichen Glauben, wonach jedes Leben begrüßenswert ist - eine Antinomie, die in zahlreichen Anti-Abtreibungsbewegungen unbeantwortet bleibt (Jentsch 2016, S. 104). Sehr aufschlussreich ist daher auch, dass sich die AfD in ihren Programmen im Zusammenhang mit ihrer Familien- und Abtreibungspolitik mit keinem Wort auf ein christliches Menschenbild bezieht, ganz anders als CDU und CSU, die sich eindeutig ,,in der Sozialethik der christlichen Kirchen“ (CDU 2007, S. 5) verorten und sich durch das ,,christliche Menschenbild [...] zum Schutz des menschlichen Lebens“ (CSU 2016, S. 4) verpflichtet sehen, was ,,auch das ungeborene Leben“ (ebd.) umfasse. Stattdessen lässt die AfD christliche Bezüge gänzlich weg und bettet ihre Bevölkerungspolitik in die Rettung der einheimischen Bevölkerung vor der Islamisierung ein. Auch ein besonderer Schutz von Frauen wird durch die vermeintlich gesteigerte Gefahr durch misogyne und hypersexualisierte junge Zuwanderer aus muslimischen Ländern begründet (vgl. Lang 2017: 71). So erfüllt die AfD in Kombination mit den „Lebensschutz“-Bewegungen zwar durchaus eine „Scharnierfunktion der radikalen Rechten zwischen Konservatismus und Rechtsextremismus“ (Birsl 2017, S. 122) und macht sich selbst anschlussfähig für christlichfundamentalistische Strömungen, verweigert aber gleichzeitig eine explizit christliche Begründung ihrer Abtreibungspolitik und beruft sich stattdessen auf einen Kulturkampf gegen eine vermeintliche inländische Überfremdung. Dies verdeutlicht eindrucksvoll die instrumentelle Beziehung, die die AfD zum Christentum pflegt. Demzufolge ist Alexander Gauland auch nicht zu widersprechen, wenn er die AfD dezidiert als keine christliche Partei bezeichnet (vgl. Althoff 2018, S. 344), zumal ein Großteil der Parteimitglieder keine religiösen Bindungen hat (vgl. Hidalgo et al. 2019, S. 3). 
Da „der Islam“ nicht als Religion per se wahrgenommen, sondern als „flexible Trope“ (Hund 2018:161) für „Fremdkultur“ und Migrationsabwehr genutzt wird, muss der Verweis auf das Christentum als Affirmation des kulturellen Antagonismus „wir“ und die „Anderen“ verstanden werden, in dem das „Eigene“ seine positive Überhöhung und somit eine Identitätsstärkung erfährt. Die Einbettung in diesen Gegensatz reicht so weit, ,dass sich sogar Konfessionslose und Atheisten in Ostdeutschland nach außen hin mit dem Christentum identifizieren, um dadurch ein vordergründiges Etikett für die Unterscheidung [...] von ,Freund ' und ,Feind ' zu gewinnen“ (vgl. Hidalgo et al. 2019, S. 4). Referenz wird eine kulturell aufgeladene, vom tatsächlichen Christsein unabhängige, religiöse Zugehörigkeit und keine echte Religiosität (vgl. Pickel 2018, S. 299). Humanismus, Aufklärung, modernes Rechtsverständnis und die Wissenschaftlichkeit werden als Errungenschaften in der „eigenen“ Kultur verortet, dem ,,andersartigen“ Islam wird unmoderne Rückständigkeit zugeschrieben, in der seine Anhänger:innen qua Abstammung verhaftet bleiben. Unterstützt wird diese Unterscheidung durch eine liberal-säkulare Rhetorik, die sich in widersprüchlicher Weise auf christliche Werte bezieht, denn sie schließt explizit das Christentum ein und den Islam aus. In der rechtspopulistischen Bezugnahme der AfD muss die Funktion des Christentums somit als ,,secularized Christianity-as-culture“ (Mouritsen 2006, S. 77) verstanden werden. Die Worthülse „Abendland“ dient als kulturüberhöhender Identifikationsstifter, der die „Kulturangst“ (Bade 2015) der Pluralisierungsgegner:innen bedient und eine funktionale Ersatzidentität liefert (vgl. Forlenza 2019, S. 139).

Daran gekoppelt lassen sich instrumentalisierende Vereinnahmungen des Judentums durch die AfD feststellen. Dies wird in der geschichtlich nicht widerspruchsfreien Semantik eines ,christlich-jüdischen“ Erbes Europas deutlich, die in der Vergangenheit auch schon von Politiker:innen anderer Parteien in Deutschland verwendet wurde und sich somit als anschlussfähig im demokratischen Diskurs erwiesen hat. Für die AfD birgt der symbolische Einschluss von Jüd:innen ins heartland primär zwei Funktionen: zum einen antimuslimische Mobilisierung und zum anderen Freisprechung vom möglichen Vorwurf rechtsextremer bzw. antisemitischer Positionen (vgl. Attia 2013). Ozan Zakariya Keskinkılıç (2019, S. 59) bezeichnet den Philosemitismus der AfD in Verbindung mit ihrer antimuslimischen Haltung im Rückgriff auf Anidjar (2003) als ,instrumentelle Dreiecksbeziehung“. Jüd:innen werden somit zu einem integralen Bestandteil der europäischen Kultur ernannt und als Opfer einer ständigen Bedrohung durch Muslim:innen dargestellt (vgl. Brubaker 2017, S. 1202). Dieser kollektive Antisemitismusvorwurf gegenüber Muslim:innen gehört zum „Einmaleins antimuslimischer Rhetorik“ (Keskinkılıç 2019, S. 58). Die Thematik findet sich auch in der Publikation der AfD Thüringen wieder: Es sei demnach eine Ausweitung des muslimischen Antisemitismus bis in die Alltagskultur hinein zu beobachten und die Verfolgung von Jüd:innen ein Merkmal des Islam, das sich direkt aus dem Koran ableiten lasse (AfD-thl 2017, S. 92f.). Die AfD Brandenburg untermauert den von ihr kolportierten Alleinherrschaftsanspruch von Muslim:innen, indem sie eine ,wachsende Hetze und Angriffe vor allem gegenüber Christen und Juden“ (AfD BB 2019, S. 58) ausmacht. Eine philosemitische Position nimmt die AfD auch ein, indem sie sich vermehrt solidarisch mit dem Staat Israel gibt. So postet die AfD Baden-Württemberg: ,dieser Staat zeigt Frau 
Merkel, wie es geht. Israel weist 40.000 illegale Flüchtlinge aus“ (AfD BW 2018). Der AfD-Landesverband Berlin postet das Bild einer Israelflagge und verbindet seine judenfreundliche Haltung direkt mit anti-linker und antimuslimischer Agitation durch die Überschrift: „ANGRIFF AUF ISRAEL-DEMO. Null Toleranz für Antisemiten. Durchsetzung gegen linke und muslimische Judenhasser nur mit der AfD“ (AfD BE 2018). Im Jahr davor hatte die AfD Berlin bereits beklagt, dass mit der „Islamisierung“ der Stadt der Antisemitismus salonfähig werde (AfD BE 2017). Alexander Gauland verband in seiner Rede im Bundestag zum 70. Jahrestag der Gründung des Staates Israel diese diskursiven Elemente, indem er feststellte, „dass die Existenzsicherung Israels am Brandenburger Tor beginnt“ (Gauland 2018) und Antisemitismus dabei nicht zum „Kollateralschaden einer falschen Flüchtlingsund Einwanderungspolitik“ (ebd.) werden dürfe. Die AfD instrumentalisiert somit die positive Darstellung eines jüdischen Erbes in Deutschland zur Agitation gegen Muslim:innen und verweist darüber hinaus realitätsfern auf die ,richtige“ Politik eines vermeintlich ethnisch homogenen jüdischen Staates Israel zur Stützung der eigenen völkischen Ideologie (vgl. Rensmann 2020, S. 328). Die Multikulturalität Israels, dessen Bevölkerung sich zu rund einem Fünftel aus arabisch-stämmigen Israelis zusammensetzt, wird in dieser Darstellung ignoriert. Dass die restriktive Asylpolitik Israels mit Menschenrechten konfligieren könnte, wird von der AfD ebenso wenig thematisiert wie dessen geopolitisch mit Deutschland unvergleichbare Situation. Der Staat Israel wird folglich ohne tiefergehende Reflektion und direktem Zusammenhang zu einem „Referenzpunkt innenpolitischer AfD-Programmatik“ (Rensmann 2020, S. 328).

Die Partei unterstellt dabei gleichzeitig dem vordergründig linken Spektrum eine Doppelmoral, da dieses aus antirassistischen Beweggründen Antisemitismus bei Muslim:innen vermeintlich verharmlose und selbst in seinem Anti-Israelismus antisemitische Haltung zeige (vgl. ebd.). „Linke und muslimische Judenhasser“ (AfD BE 2018) werden gleichgesetzt, Antisemitismus von anderen Gruppierungen ausgeklammert. Obgleich auch von linken Kreisen unterstützte Bewegungen, bspw. die BDS-Kampagne, nicht von Antisemitismus-Vorwürfen freizusprechen sind, zeigt die AfD somit selbst eine Doppelmoral: Denn antisemitische Haltungen sind in den eigenen Reihen weit verbreitet (vgl. ebd.), auch wenn sie in den Anfangsjahren hinter den Themen Islam, Migration und nationale Identität eher zurücktraten (vgl. Grimm und Kahmann 2017, S. 41). Samuel Salzborn sieht die AfD gar auf dem Weg „von einer Partei für Antisemiten zu einer antisemitischen Partei“ (Salzborn 2019, S. 197), da sie zwar programmatisch keine expliziten Anknüpfungspunkte für Antisemit:innen biete, ihr indifferenter Umgang mit implizit und explizit judenfeindlichen Positionierungen sie dennoch dafür anschlussfähig mache.

Generell lassen sich im Weltbild der AfD, das sich aus einem völkisch exklusiven Nationalismus als Gemeinschaftskonstruktion und der Vorstellung einer vermeintlich korrupten Elite als „Volksfeinde“ speist, camouflierte und codierte Tropen eines modernisierten Antisemitismus wiederfinden (vgl. Rensmann 2020, S. 321). Die Vorstellung eines auf vorpolitischen Kriterien basierenden, ethnisch homogen fantasierten Kollektivs der „Deutschen“ schließt an völkische Konzepte des Nationalsozialismus an, die als Grundlage für Antisemitismus verstanden werden müssen (vgl. Salzborn 2019, S. 211). Die AfD Sachsen-Anhalt versuchte bereits 2015, den Begriff 
der „Volksgemeinschaft“ zu enttabuisieren, als sie auf Facebook ihre Weihnachtsgrüße mit dem Appell verband, sich Gedanken , über [...] Verantwortung für die Volksgemeinschaft" (zit. n. Gensing 2015) zu machen. Auch die ehemalige Parteichefin Frauke Petry betonte 2016, der Begriff ,,völkisch“ müsse wieder positiv aufgeladen werden (vgl. Balzli und Kamann 2016). Während sich der völkische Nationalismus der Partei nicht nur gegen Jüd:innen richtet, sondern auch weitere Menschen aus der nationalen Gemeinschaft ausschließt, lassen sich im Antagonismus „Volk“ vs. „Elite“ konkret antisemitische Figurationen nachweisen. Die ,,in Verschwörungen verwickelte“, „global vernetzte“, ,korrupte“ und „,kosmopolitische“ „Elite“, die angeblich das nationale Volk ,verrät“, ist eine Imago, die dem Arsenal des modernen Antisemitismus entstammt und anschlussfähig an antisemitische Verschwörungsmythen ist (vgl. Rensmann 2020, S. 316). Aufgrund bereits vorhandener kulturellideologischer Deutungsmuster erfolgt die Assoziation der als „Globalisten“ bezeichneten ,kosmopolitischen“ Elite mit einer jüdischen Weltverschwörung bei den Anhänger:innen der Partei ohne große Umwege. Vor allem im Bedrohungsszenario des u. a. von Björn Höcke kolportierten Bevölkerungsaustausches lassen sich Bezugnahmen auf jüdische „Strippenzieher“ als Kollaborateur:innen finden. So bezeichnete Höcke die EU als „Globalisierungsagentur“, die den ,als pervers zu bezeichnenden Geist eines George Soros“ (zit. n. Siemens et al. 2020) exekutiere, und der deutsche Verfassungsschutz sei laut Höcke ,zum reinen Exekutivorgan für den völkerauflösenden (...) Geist eines George Soros geworden“ (zit. n. Mascolo et al. 2019). Der jüdische Finanzier Soros ist Teil rechtsextremer Verschwörungsmythen, die ihn als „Drahtzieher“" globaler Veränderungen, vor allem Migrationsbewegungen, darstellen und diffamieren. Der AfD-Bundestagsabgeordnete Thomas Seitz bezeichnete Aktivist:innen von Fridays For Future als ,Soros-Jugend“ (zit. n. Schwarz 2020, S. 70), was eine jüdische „Steuerung“ der Demonstrierenden suggeriert. Im Hinblick auf diese Beispiele hält Rensmann fest, dass Antisemitismus hier implizit als kultureller Code (vgl. Volkov 2000) und symbolisches Bindemittel zur Ablehnung moderner Pluralisierungsprozesse fungiere (vgl. Rensmann 2020, S. 317).

Eng mit der nativistischen Positionierung der AfD verknüpft lässt sich ein ,,sekundärer Antisemitismus aus Erinnerungsabwehr“" (vgl. ebd.: 323) beobachten, der mit einem Geschichtsrevisionismus und dem Wunsch nach kollektiver Unschuld in Bezug auf die NS-Vergangenheit einhergeht (vgl. Salzborn 2019, S. 202). Salzborn macht in der Partei ein ,geradezu besessenes“ (ebd.: 203) Verhältnis zum Nationalsozialismus aus, dessen geschichtsrevisionistische Implikation lange Zeit kaschiert wurde. In jüngster Vergangenheit trat es allerdings immer häufiger zutage. So möchte die AfD laut Grundsatzprogramm die ,aktuelle Verengung der deutschen Erinnerungskultur auf die Zeit des Nationalsozialismus“ aufbrechen, um zu einer erweiterten Geschichtsbetrachtung zu gelangen, ,die auch die positiven, identitätsstiftenden Aspekte deutscher Geschichte mit umfasst“" (AfD 2016, S. 48). Dabei geht es letztlich um die Abwehr von empfundener Minderwertigkeit und Schuld, die von AfD-Akteur:innen gleichsam mit der Übernahme des „Opfermythos“ betrieben wird (vgl. Salzborn 2019, S. 205). Dies wird deutlich, wenn Alexander Gauland betont, dass andere „Völker“ ihre Identität sehr viel stärker verteidigen dürften, während Auschwitz als Symbol ,viel in uns“ (Gauland 2016) zerstört und Hitler ,,den Deutschen“ das Rückgrat gebrochen habe. Björn Höcke kritisierte den vermeintlichen 
Opfermythos in rechtsextremer Manier, als er bei einer Veranstaltung der „Jungen Alternative" die Bombardierung Dresdens im zweiten Weltkrieg durch die Alliierten als „Kriegsverbrechen“, dessen eigene Opfer man bis heute nicht in der Lage sei zu betrauern (vgl. DLF 2017), bezeichnete. Das Holocaust-Mahnmal in Berlin nannte er ein „Denkmal der Schande“ (ebd.). Es sei daher eine „,erinnerungspolitische Wende um 180 Grad“ (ebd.) nötig. Gaulands und Höckes Aussagen illustrieren die geschichtsrevisionistischen Ambitionen der Partei, einen Schlussstrich unter die christlich-europäische und deutsche Gewalt gegen Jüd:innen zu ziehen (vgl. Attia 2018, S. 31), um einen völkischen Patriotismus wiederzubeleben. Dabei werden die NS-Vergangenheit und der Holocaust relativiert und zugleich antisemitische Positionierungen deutlich.

Antisemitismus lässt sich aber auch ohne Umwegkommunikation in expliziten Äußerungen von Parteimitgliedern und Parteiverbänden der AfD und dem Umgang damit ausmachen. Öffentliche Aufmerksamkeit erhielt vor allem der Fall des ehemaligen Landtagsabgeordneten Wolfgang Gedeon, der sich in seinen Schriften eindeutig antisemitisch geäußert hatte. So behauptet er etwa, dass ,die Juden“ ,,an der Versklavung der Menschheit"“ (zit. n. Saure und Maegerle 2016) arbeiteten, mit dem Ziel einer „Judaisierung der christlichen Religion und Zionisierung der westlichen Politik“ (zit. n. Bender und Soldt 2016). Die Partei reagierte nicht mit einem sofortigen Ausschluss, sondern versuchte, die Beurteilung, ob diese Aussagen antisemitisch seien, durch die Abwälzung auf außerparteiliche Gutachter:innen zu externalisieren (vgl. Salzborn 2019, S. 208f.). Somit distanziert sich die AfD davon, Antisemitismus in den eigenen Reihen selbst be- und verurteilen zu können, um Mitglieder mit antisemitischen Haltungen nicht vor den Kopf zu stoßen. Bezeichnend dafür war in der Folge die fehlende Mehrheit in der Fraktion beim später eingeleiteten Ausschlussverfahren Gedeons, der letztlich freiwillig aus der Partei austrat (vgl. Grimm und Kahmann 2017, S. 41). Eine Distanzierung der Partei blieb aus (vgl. ebd.: 45). Neben der „Causa Gedeon“ finden sich weitere offen antisemitische Äußerungen von Mitgliedern der Partei (vgl. dazu Salzborn 2019, S. 209f.; Rensmann 2020, S. 325ff.), die die Einschätzung eines instrumentellen Charakters der philosemitischen und pro-israelischen Propaganda nachweislich stützen. Laut der LAS wiesen 2020 die Befragten mit der Wahlpräferenz AfD mit Abstand den größten Anteil an Personen mit antisemitischen Einstellungen auf (vgl. Kiess et al. 2020, S. 240).

Somit lässt sich festhalten: Die Anrufung eines ,,christlich-jüdischen Abendlandes“ zielt in der AfD darauf ab, antisemitische Haltungen von sich zu weisen, und verortet Antisemitismus in migrantischen - vornehmlich muslimischen - Milieus. Dabei wird die lange Geschichte des christlichen Antijudaismus und Antisemitismus ignoriert, wie die jüdische Philosophin Almut Shulamit Bruckstein Çoruh (2010) deutlich macht: „Nein, es gab keine jüdisch-christliche Tradition, sie ist eine Erfindung der europäischen Moderne und ein Lieblingskind der traumatisierten Deutschen." Schlussstrich-Forderungen und Holocaust-Relativierungen zeigen antisemitische Züge und dienen - genauso wie israelfreundliche Positionierungen der Stärkung des völkischen Konstrukts eines heartlands. Antisemitismuskritik wird zum Zwecke antimuslimischer Mobilisierung und Migrationsabwehr instrumentalisiert. Jüd:innen dienen dann als ,,instrumentelle Kontrastfolie“ (Keskinkılıç 2019, S. 64) für die Agitation der AfD. Analog zum Islam erweist sich die Bezugnah- 
me zu Christentum und Judentum nicht primär als religiöser Werterahmen, sondern vielmehr als die Verklausulierung nativistischer rechtsextremer Positionen in der Partei, die klare Zugehörigkeiten zum heartland durch antagonistische und vergemeinschaftende Differenzmarkierungen festlegen. Dabei, so soll im nächsten Teil argumentiert werden, handelt es sich um eine bewusste Diskursivierung religiöser Zuschreibungen, um die eigenen rechtsextremen Positionen bestmöglich mit denen potenzieller Wähler:innen in Einklang zu bringen und somit im Sinne eines VoteSeekings Stimmen zu maximieren.

\section{Antimuslimische Hetze als Vote-Seeking-Strategie der AfD}

Es hat sich gezeigt, dass Verweise auf das „christlich-jüdische Abendland“ durch die AfD in vielerlei Hinsicht widersprüchlich sind und sich vor allem gegen den Islam und Muslim:innen richten. Doch welche Funktion hat diese Umwegkommunikation? Die religiöse Bezugnahme der AfD soll im Folgenden als bewusste Agitation zur Stimmenmaximierung untersucht werden. Ausgehend von Wolfgang C. Müller und Kaare Strøm (2010, S. 5) verfolgen politische Parteien grundsätzlich drei mehr oder weniger stark gewichtete Ziele: die Regierungsbeteiligung (officeseeking), die Durchsetzung politischer Ziele (policy-seeking) und die Stimmenmaximierung bei Wahlen (vote-seeking). Demnach koalieren Parteien, die stärker auf eine Regierungsbeteiligung abzielen, mitunter auch mit ideologisch ihnen eher ferneren Parteien, während stärker Policy-orientierte Parteien tendenziell mit inhaltlich verwandteren Parteien in eine Regierung eintreten. Freilich treten die einzelnen Ziele nicht isoliert voneinander auf. Sie beeinflussen sich vielmehr wechselseitig und gehen bisweilen miteinander einher.

Rechtspopulistische Parteien stehen vor dem Problem, dass ihre Anti-Parteienund Anti-Establishment-Haltung dem aufkommenden Zwang, sich zu institutionalisieren, mitunter widerspricht, weshalb sie an Glaubwürdigkeit verlieren können (vgl. Taggart 2004, S. 276). Dies ist ein Effekt, der sich mit einer Regierungsübernahme und der nahezu zwangsläufig damit verbundenen Koalition mit einer etablierten Partei weiter verschärfen kann, wenngleich die vielfach erhoffte Entzauberung der Rechtspopulist:innen in Regierungsverantwortung bislang kaum langfristig beobachtet werden konnte. Einerseits ist eine politische Institutionalisierung also quasi unabdingbar, um die politische Agenda langfristig mitzubestimmen (vgl. Taggart 2002, S. 79). Andererseits sind rechtspopulistische Parteien häufig einem Cordon sanitaire ausgesetzt, was bedeutet, dass alle etablierten Parteien eine Koalition mit ihnen mehr oder weniger kategorisch ausschließen. Während sich Parteien wie die FPÖ oder die Lega per Salvini (ehemals: Lega Nord) aus diesem Zustand, unter anderem durch das Druckmittel eines sehr hohen Wähler:innenzuspruchs, befreien konnten, behält er für die AfD bisher Gültigkeit. Folglich kann davon ausgegangen werden, dass die AfD vor allem Vote-Seeking- und Policy-Seeking-Strategien verfolgt, da derzeit weder eine Koalition noch eine absolute Mehrheit als realistische Ziele angesehen werden können. Dahingehend äußerte sich auch Alexander Gauland vor der Bundestagswahl 2017, der größeres Potenzial für die Partei in der Opposition sah (vgl. Spiegel online 2016). Ohne die Chance auf Regierungsverantwortung 
lässt sich die Agenda vor allem durch die Generierung öffentlicher Aufmerksamkeit bestimmen. Dabei birgt die häufig eingenommene Fundamentalopposition auch Chancen: Man kann sich glaubwürdig als Gegnerin des Establishments positionieren und muss keine Rücksicht auf mögliche Koalitionspartner:innen nehmen.

Gemäß der Salienztheorie versuchen Parteien, die Aufmerksamkeit auf jene Themen zu lenken, bei denen sie am meisten Potenzial für sich selbst sehen, anstatt über jedes Politikfeld öffentlich zu diskutieren (vgl. Dolezal et al. 2014, S. 59). Meyer und Wagner (2013, S. 1264) zeigen in einer Studie aus 22 Ländern, dass Parteien ihre Kernthemen durchaus auf Basis von strategischen Vote-Seeking-Überlegungen auswählen. Am häufigsten sind strategische Wechsel bei jüngeren Parteien aus der Opposition nachzuweisen (vgl. ebd.: 1248). Für rechtspopulistische Parteien birgt vor allem die Politisierung neuer Themen, allen voran die Migrationsfrage, ein großes Potenzial zur Profilierung und Stimmenmaximierung (vgl. Rydgren 2005, S. 422). Als wirksamstes Thema in diesem Kontext erweist sich die gezeigte Islamisierung des Diskurses. Langfristig lassen sich der Druck auf andere Parteien aus der Opposition heraus und die Bestimmung öffentlicher Debatten als Agenda-Setter allerdings nur mit gewissen Wahlerfolgen aufrechterhalten. Im Folgenden soll daher die These belegt werden, wonach es sich bei der antimuslimischen Propaganda und dem Verweis auf ein ,jüdisch-christliches“ Erbe der AfD nicht per se um eine ideologische Überzeugung, sondern auch um eine gezielte Vote-Seeking-Strategie handelt. Denn die Partei mag aus ihrem nativistischen Weltbild heraus ein Interesse an einem homogenen Nationalstaat haben, dass sie aber vor allem den Islam und damit verbunden das Christentum und das Judentum ins Zentrum der eigenen Agenda rückt, ist der erhöhten Anschlussfähigkeit in der Bevölkerung geschuldet.

Die historische Entwicklung der AfD kann zur Unterstützung dieser These herangezogen werden. Die Partei konnte von großen gesellschaftlichen Krisen profitieren, indem sie auf Responsivitätsdefizite im Parteiensystem reagierte. Die einheitliche Politik in der Eurokrise schuf „ein Gelegenheitsfenster für populistische Akteure“ (Bebnowski 2015, S. 33). Sie stellte damit den Gründungsanlass der AfD dar. Die erweiterte Gründungsursache ist in einer allgemeinen Unzufriedenheit über die Positionen von Union und FDP zu suchen, wodurch sich eine Repräsentationslücke in der Parteienlandschaft auftat (vgl. Franzmann 2014, S. 115). Die Eurokrise war in den Augen der Bevölkerung das wichtigste politische Problem zwischen 2011 und 2013 (vgl. Giebler et al. 2015, S. 138), und die AfD war in der öffentlichen Wahrnehmung die einzige Partei, die merklich von den Positionen des politischen Mainstreams in Deutschland abwich, was ihren rasanten Aufstieg bewirkte. Es lässt sich also durchaus glaubhaft argumentieren, dass die AfD nicht als genuin rechtspopulistische Partei gegründet wurde, was auch die damalige wissenschaftliche Auseinandersetzung nahelegt. Für diese Annahme spricht ebenfalls, dass mit der Abwahl von Bernd Lucke und der stetigen Öffnung nach Rechtsaußen ein beträchtlicher Teil des konservativen Gründungszirkels, der den offen rechtspopulistischen und antimuslimischen Kurs nicht mittragen wollte, aus der Partei austrat (Pfahl-Traughber 2019, S. 6). Gleichwohl ist nicht zu verkennen, dass sich auch Parteigründer Lucke des Potentials am rechten Rand bewusst war und sich, zumindest aus wahltaktischen Gründen, nie völlig von rechten Positionen distanzierte, im Gegenteil, sich ihnen sogar durch ein Thesenpapier zum Islam annäherte (vgl. Lucke 2013). Bereits 2013 forderte die 
Partei eine Reform des Einwanderungsrechts zugunsten qualifizierter Zuwanderung, allerdings noch ohne Bezugnahme auf ,nationale Einheit“ oder „Kultur“ (vgl. AfD 2013, S. 4). Außerdem solidarisierte man sich früh mit Teilen von Pegida, da man thematische Überschneidungen und mitunter auch einen natürlichen Verbündeten sah (vgl. Grabow 2016: 174). Schon 2014 zeigte sich, dass die Eurokrise weder das bestimmende öffentliche Thema noch das Hauptmotiv einer Wahlmotivation für die AfD geblieben war. Vielmehr konnte eine kritische Haltung gegenüber Zuwanderung und „den Eliten“ als einigendes Merkmal der AfD-Wähler:innen ausgemacht werden (vgl. Häusler und Roeser 2014, S. 45). Die Partei wurde gewissermaßen zu einem demokratisch legitimierten Ventil für Bevölkerungsteile mit rechtsextremen und muslimfeindlichen Einstellungsmustern, die sich von den Aussagen Sarrazins angesprochen fühlten und bis dahin auf eine erfolgreiche Entsprechung im Parteiensystem gewartet hatten. Diesen Tendenzen duldete die AfD zunächst, doch spätestens seit der Übernahme des Vorsitzes durch Frauke Petry und den ersten Landtagswahlen in Ostdeutschland trug sie zu ihrer aktiven Verbreitung bei. Der AfD gelang es, ,,politisch heimatlos gewordenen Nationalkonservativen sowie neurechten Kräften und früheren Aktivisten rechtspopulistischer Kleinstparteien ein neues parteipolitisches Dach“ (Häusler und Roeser 2015, S. 8) zu bieten. Besonders im Osten der Republik setzten sich mit Björn Höcke und Andreas Kalbitz offen rechtsextreme Akteure an die Spitze der Landesverbände, wodurch es nur eine logische Konsequenz war, dass Frauke Petry, die zuvor selbst ein Förderin des Rechtsrucks gewesen war, einem weiteren Rechtsruck weichen musste. Sie ist dabei auch über den Versuch gestolpert, die Partei zugunsten einer möglichen Koalition mit der Union oder der FDP zu mäßigen (vgl. Pfahl-Traughber 2019, S. 6). Die Durchsetzung ihrer Gegner:innen zeigt die strategische Präferenz der Partei auf und bekräftigt somit die These einer stärker auf Vote- und Policy-Seeking ausgerichteten Strategie. Die Erfolglosigkeit von Luckes und Petrys Nachfolgeparteien beweisen indes eindrucksvoll, dass explizit keine Nachfrage nach einer konservativeren, aber gemäßigten Alternative zur Union am rechten Rand besteht.

Die größten Wahlerfolge und Zuwächse erreichte die AfD im Zuge der sogenannten „Flüchtlingskrise“ von 2015. Neben der allgemeinen Thematisierung und Problematisierung von Migration ist dabei ein zunehmender Fokus auf das Thema Islam zu beobachten. Geleakte interne Besprechungen offenbaren die taktischen Überlegungen von Beatrix von Storch, die sich eine besonders hohe Aufmerksamkeit für die Partei durch die Fokussierung des Programms auf den Islam versprach, und das Thema deshalb für besonders hilfreich hielt (vgl. Biskamp 2018, S. 254). Auch dies zeigt, dass die Islamisierung gesellschaftlicher Probleme neben der zweifelsohne vorhandenen Ideologie eine bewusste Entscheidung zur Steigerung der öffentlichen Aufmerksamkeit und damit letztlich auch der Stimmenmaximierung ist. Anders als mit offensichtlich rassistischen Themen lässt sich durch die chiffrierte Agitation gegen Muslim:innen eine demokratische Gesinnung aufrechterhalten. Deshalb muss das Amalgam aus Philosemitismus und aus dem vordergründigen Einsetzen für Rechte von Frauen und Homosexuellen sowie für liberale Werte einerseits und die dem gegenübergestellte Skizzierung der Gefahr einer islamischen Bedrohung andererseits als wahltaktisches Manöver zur Akzeptanzgewinnung gewertet werden (vgl. Brubaker 2017, S. 1203). Ein Blick auf die Einstellungsmerkmale der AfD- 
Wähler:innen untermauert diese These. Während nur etwas mehr als ein Viertel des Elektorats rechtsextremistische Einstellungen aufweist, was zwar deutlich über dem bundesdeutschen Schnitt liegt, aber bei Weitem keine Mehrheit bildet, ordnen sich $44 \%$ der Wähler:innen der politischen Mitte und 14\% gar dem linken Spektrum zu (vgl. Hambauer und Mays 2018, S. 137). Auch wenn die Tendenz innerhalb des Elektorats stetig nach rechtsaußen geht (vgl. Celik et al. 2020, S. 164), so zeigt sich doch Folgendes: Offensichtlich werden die Positionen der AfD nicht in einem Maße als rechtsextrem wahrgenommen, dass sie eine Wahl für Menschen, die sich selbst nicht als rechts(extrem) einstufen, ausschließen. Abseits der persönlichen Einschätzung sind sich AfD-Wähler:innen nahezu widerspruchslos einig in ihrer Ablehnung der Flüchtlingspolitik von Angela Merkel, in der Sorge vor zunehmender Kriminalität durch Zuwanderung und auch in der Angst vor einem zunehmenden Einfluss des Islam (vgl. Hofrichter und Niedermayer 2016, S. 276). Anti-muslimische Einstellungen bilden hierbei einen, um nicht zu sagen, den Schlüssel für die hohen Zustimmungsraten der AfD (vgl. Huber und Yendell 2019, S. 66). Dabei bleibt die Angst vor der Zuwanderung nicht auf Menschen beschränkt, die sich vor einer Konkurrenzsituation auf dem Arbeitsmarkt fürchten, vielmehr hat man es hier mit einem generellen „Gefühl der kulturellen Bedrohung durch die Zuwanderung von ethnischkulturell fremden Menschen nach Deutschland“ (Lengfeld 2018, S. 306) zu tun. In deutlich größerem Ausmaß als wirtschaftliche Bedrohungen spielen also individuelle und kollektive Verlustängste der Kultur eine Rolle (vgl. ebd.: 307). Die AfD trifft hier auf einen echten Wähler:innenmarkt, der von keiner anderen Partei bedient wird. Es ist kein Zufall, dass die LAS seit 2014 eine Zunahme in den antimuslimischen Haltungen der Bevölkerung feststellt, also in dem Zeitraum, in den auch die Hinwendung der AfD zu diesem Thema fällt (vgl. Celik et al. 2020, S. 165). Ihr Konzept funktioniert, weil ihre Verklausulierung in vielen Teilen der Bevölkerung eben gerade nicht als rechtsextrem und rassistisch wahrgenommen wird. Vielmehr ist antimuslimischer Rassismus bis weit in den gesellschaftlichen Mainstream vorgedrungen. Ruth Wodak (2018, S. 323) beschreibt dies im Hinblick auf Österreich als eine „schamlose Enttabuisierung“, die zu einer Normalisierung rechtspopulistischer Themen geführt habe, weshalb nun auch Parteien wie die ÖVP versuchen würden, Wähler:innen in diesem Segment zu gewinnen. Dabei ist der Rückgriff auf bestehende Vorurteile in der Bevölkerung eine wichtige Erfolgsvoraussetzung der AfD. Floris Biskamp (2018, S. 264) weist aber zurecht darauf hin, dass bestehende Vorurteile alleine den Fokus auf den Islam nicht erklären können, schließlich bestünden ähnlich große negative Einstellungen auch gegenüber anderen Gruppen, wichtig seien hingegen auch die Skandalisierung und Aufmerksamkeitsgenerierung.

Es soll hier veranschaulicht werden, dass anti-muslimische Einstellungen gewissermaßen den perfekten Anknüpfungspunkt an das Wähler:innenpotential bieten. Anders als der Antiziganismus bzw. Gadjé-Rassismus, der auf noch größere Zustimmungswerte in der Bevölkerung käme (vgl. Decker et al. 2020, S. 66), haben Muslim:innen bzw. Menschen, die aufgrund phänotypischer und anderer Merkmale als Muslim:innen gelesen werden, eine deutlich höhere Sichtbarkeit in der Bevölkerung. Diese wird durch die AfD weiter erhöht, indem sie, jenseits der tatsächlichen Wahrheit, die Erzählung „Migrant= Flüchtling= Muslim“ prägt. Hinzu kommt eine ohnehin schon aufgeladene Debatte, wie beispielsweise der Aufruhr um den ehema- 
ligen Bundespräsidenten Christian Wulff (CDU) verdeutlicht, als er den Islam als Teil von Deutschland anerkannte. Gleichzeitig unterliegen antimuslimische Haltungen eben nicht jener Stigmatisierung, wie es beim Antisemitismus der Fall ist. Ganz im Gegenteil konstruiert man durch die Integration des Judentums in das ,christlichjüdische Abendland“, über die Solidarisierung mit Israel und den Verweis auf den vermeintlich inhärenten Antisemitismus von Muslim:innen eine zusätzliche Ebene des demokratischen Scheins. Anti-muslimische Haltungen werden in die angebliche Verteidigung liberaler Werte eingebettet und mit dem eigenen Philosemitismus untermauert (vgl. Weiland 2016) Dass es sich dabei nur um einen äußeren Anstrich handelt und antisemitische Positionen in der AfD weit verbreitet sind, konnte im vorangegangenen Kapitel gezeigt werden und entlarvt das taktische Kalkül hinter dem Agenda-Setting. Ähnlich wie die vordergründige Freundschaft zum Judentum ist schließlich auch die Berufung auf das Christentum als taktisches Mittel zu verstehen. Wie zuvor gezeigt wurde, ist weder bei den Wähler:innen noch in der Programmatik der Partei eine tiefergehende christliche Einfärbung zu erkennen. Zwar gibt es auf der einen Seite eine gewisse Nähe zu ultrakonservativen Kreisen, auf der anderen Seite bestehen jedoch diametrale Gegensätze zum christlichen Weltbild. Der Religionsbezug hilft dennoch, das „Sprengen verfassungsrechtlicher Grenzen der demokratischen Verfügungsgewalt und somit das Einreißen aller Hemmnisse für eine Tyrannei der Mehrheit“" (Hidalgo 2018, S. 186) sowie die grundsätzliche Pluralismusfeindlichkeit zu kaschieren und sich trotz der rechtsextremen Haltungen nicht ins Aus manövrieren zu lassen. So ,werden gezielt solche Vorurteile bedient, die eher sozial akzeptiert sind, um potenzielle Wähler - die ja gerade keine überzeugten Rechtsextremisten sind - nicht zu verschrecken“ (Heitmeyer und Klein 2012, S. 91).

Aus diesen Zusammenhängen heraus lässt sich schließlich das Interesse der AfD an einer zuspitzenden Islamisierung des Diskurses verdeutlichen. Einerseits profitiert die Partei von ihrem Alleinstellungsmerkmal als Vertreterin muslimkritischer bis -feindlicher Haltungen, die in der Bevölkerung großen - wenn auch nicht mehrheitlichen - Anklang finden, andererseits schafft sie es, im Namen von liberalen Werten und Religionsfreiheit eben jene liberalen Werte und die Religionsfreiheit mit Füßen zu treten, ohne dass es ihr von Sympathisant:innen und Anhänger:innen als rechtsextreme Haltung ausgelegt wird. Die Verengung auf diese Thematik muss, ob der offensichtlichen Überbewertung in der öffentlichen Wahrnehmung, zweifellos als Taktik der Stimmenmaximierung in bestimmten Wähler:innenkreisen bewertet werden.

\section{Fazit}

Es lässt sich festhalten, dass die rechtspopulistische AfD den Bezugsrahmen ihrer Agenda neben der weiterhin stark völkisch-nationalen Akzentuierung häufig mit dem Verweis auf christliche, jüdische und ,abendländische“ Werte verbindet. Die Ausgangsthese legte hierbei nahe, dass es sich beim (einseitigen) „Schulterschluss" der AfD mit dem Christentum und mit dem Judentum nicht um ein religiöses Wertebekenntnis handelt, sondern die Partei sich in populistischer Manier eine Stimmenmaximierung verspricht. Dazu instrumentalisiert sie religiöse Bezüge als 
identifikationsstiftende Gegenposition zum gesellschaftlich verbreiteten Feindbild des Islam. Dieses Feindbild, so zeigt die Geschichte, ist in der Bundesrepublik nicht neu und lässt sich primär auf einen Wandel ethno-natio-kultureller Differenzlinien im Zuge eines veränderten nationalen Selbstverständnisses und der Selbstbeschreibung als Einwanderungsland zurückführen. Aus Migrant:innen wurden in der Folge zunehmend Muslim:innen gemacht, um Demarkationslinien aufrechtzuerhalten und pluralitätsfeindlichen Gesinnungen einen figurativen Gegenspieler zu liefern. Die AfD greift diese kulturellen Verlust- und Pluralisierungsängste auf und adaptiert das „Feindbild“ der Muslim:innen als Outgroup. Dabei schließt sie an altbekannte Muster des bundesdeutschen „Ausländer“-Diskurses an. Religion spielt hierbei nur eine ostentative Rolle. Die AfD stellt die Religion Islam als Kultur und politische Ideologie dar, der sich deren Anhänger:innen qua Geburt nicht entziehen können, und verweist somit auf die vermeintlich dringende Notwendigkeit einer Einschränkung religiöser Rechte von Muslim:innen. Gleichzeitig formt sie im Rückgriff auf den Grenzmarker Kultur einen dichotomen Antagonismus des ,wir“ (Deutsche) und die „Anderen“ (Muslim:innen). „Der Islam“ wird dabei als monolithischer Block dargestellt, dem Säkularisierungs- und Pluralisierungsprozesse abgesprochen werden (vgl. Ceylan 2017, S. 85). Die Abgrenzung erfolgt vor allem über rassistische Zuschreibungen. All dies geschieht unter dem Schutzschild der Islamkritik, die sich die AfD ,als Teil des Grundrechts der freien Meinungsäußerung“ (AfD 2016, S. 96) nicht nehmen lassen will, obgleich sie in vielen Forderungen rassistisch, demokratiefeindlich und rechtsextrem ist und somit selbst Grundrechte missachtet. Die AfD verbindet so antimuslimische Hetze mit (rechts-)populistischer Agitation, weshalb Häusler (2019) auch von „Antimuslimischem Populismus“ spricht.

Neben der dominanten Rolle, die der Islam und Muslim:innen in der antipluralistischen Vote-Seeking-Strategie der AfD annimmt, lässt sich auch die Bezugnahme auf das Christentum und das Judentum als opportunistisches Instrumentarium des Wähler:innenfangs kenntlich machen. Die philosemitischen Bekundungen der AfD erfolgen mit dem Ziel, antimuslimische Agitationen mit dem Generalverdacht eines genuin muslimischen Antisemitismus zu untermauern. Gleichzeitig erfolgt eine Freisprechung von antisemitischen Anklagen, die aufgrund geschichtsrevisionistischer und explizit judenfeindlicher Äußerungen offen rechtsextremer Mitglieder gegen die Partei erhoben werden. Sympathiebekundungen zum als ethnisch homogen dargestellten ,jüdischen“ Staat Israel dienen unter dem Strich der Stärkung der eigenen nativistischen Haltungen und können daher keineswegs mit „Toleranz" gegenüber jüdischem Leben in Deutschland gleichgesetzt werden. Verweise auf das Christentum nutzt die AfD ebenfalls zur Bekräftigung antimuslimischer Mobilisierung, indem sie den orientalistischen Antagonismus „Orient“ vs. „Okzident“ bedient, der die Kultur von Muslim:innen als unveränderlich und unvereinbar mit liberal-säkularen Werten Europas festschreibt. Mit diesem „religious turn“ (Forlenza 2019, S. 35) folgt die AfD einem Trend unter Europas Rechtspopulist:innen. Das europäische Christentum, das dem Judentum trotz gegenläufiger Geschichte „,solidarisch“ zur Seite gestellt wird, erfährt eine kulturelle Aufladung und wird mit dem Geist der Aufklärung und der Trennung von Staat und Kirche assoziiert, die im politisierten Islam undenkbar sei. Mit der Betonung der Säkularisierung manifestiert die AfD ihre ablehnende Haltung gegenüber den großen Kirchen, die losgelöst von ihrem Bild des 
Christlichen zu betrachten sind. Das „,christlich-jüdische Abendland“ wird dadurch sowohl zum imaginierten retrospektiven Sehnsuchtsort eines Europas vor der Massenzuwanderung als auch zur schutzbedürftigen Festung gegen die Islamisierung erhoben. Es handelt sich demnach um religiöse Verklausulierungen nativistischer Positionen, die sich in der Partei selbst und - das zeigen jüngste Studien - auch im Elektorat der AfD wiederfinden lassen und daher enormes Wähler:innenpotenzial mit sich bringen (vgl. Pesthy et al. 2020). Der Religionsbezug dient daher letztlich der eigenen Entdämonisierung: Denn Christ:innen und Jüd:innenfreund:innen seien keine Rechtsextremist:innen und übten letztendlich nur ,legitime Islam-Kritik“.

Funding Open Access funding enabled and organized by Projekt DEAL.

Open Access Dieser Artikel wird unter der Creative Commons Namensnennung 4.0 International Lizenz veröffentlicht, welche die Nutzung, Vervielfältigung, Bearbeitung, Verbreitung und Wiedergabe in jeglichem Medium und Format erlaubt, sofern Sie den/die ursprünglichen Autor(en) und die Quelle ordnungsgemäß nennen, einen Link zur Creative Commons Lizenz beifügen und angeben, ob Änderungen vorgenommen wurden.

Die in diesem Artikel enthaltenen Bilder und sonstiges Drittmaterial unterliegen ebenfalls der genannten Creative Commons Lizenz, sofern sich aus der Abbildungslegende nichts anderes ergibt. Sofern das betreffende Material nicht unter der genannten Creative Commons Lizenz steht und die betreffende Handlung nicht nach gesetzlichen Vorschriften erlaubt ist, ist für die oben aufgeführten Weiterverwendungen des Materials die Einwilligung des jeweiligen Rechteinhabers einzuholen.

Weitere Details zur Lizenz entnehmen Sie bitte der Lizenzinformation auf http://creativecommons.org/ licenses/by/4.0/deed.de.

\section{Literatur}

\section{Verwendete Literatur}

Althoff, Andrea. 2018. Right-wing populism and religion in Germany: conservative Christians and the Alternative for Germany (AfD). Zeitschrift für Religion, Gesellschaft und Politik 2(2):335-363.

Amir-Moazami, Shirin. 2009. Islam und Geschlecht unter liberal-säkularer Regierungsführung. Die Deutsche Islam Konferenz. In Juden und Muslime in Deutschland. Recht, Religion, Identität, Hrsg. José Brunner, Shai Lavi, 186-205. Göttingen: Wallstein.

Anidjar, Gil. 2003. The Jew, the Arab. A history of the enemy. Stanford: Stanford University.

Attia, Iman. 2009. Die „westliche Kultur“ und ihr Anderes: Zur Dekonstruktion von Orientalismus und antimuslimischem Rassismus. Bielefeld: transcript.

Attia, Iman. 2013. Privilegien sichern, nationale Identität revitalisieren: Gesellschafts- und handlungstheoretische Dimensionen der Theorie des antimuslimischen Rassismus im Unterschied zu Modellen von Islamophobie und Islamfeindlichkeit. Journal für Psychologie 21(1):1-30.

Attia, Iman. 2015. Die Religion und Kultur der Anderen: Zur Entsorgung historischer, gesellschaftlicher und politischer Dimensionen im Islamdiskurs. In Das Unbehagen an der Kultur, Hrsg. Ingo Schneider, Martin Sexl, 181-199. Hamburg: Argument.

Attia, Iman. 2018. Den Rassimus gibt es nicht: Zum Verhältnis von Antisemitismus und antimuslimischem Rassismus. In Fremdgemacht et Reorientiert. Jüdisch-muslimische Verflechtungen, Hrsg. Ozan Zakariya Keskinkılıç, Ármin Langer, 21-44. Berlin: Yilmaz-Günay.

Bade, Klaus J. 2015. Kulturvielfalt, Kulturangst und Negative Integration in der Einwanderungsgesellschaft. In Migration - Religion - Identität. Aspekte transkultureller Prozesse, Hrsg. Kerstin Kazzazi, Angela Treiber, 1-34. Wiesbaden: Springer VS.

Baier, Alicia. 2019. Die Entmündigung der Frau. https://www.gwi-boell.de/de/2019/09/19/die-entmuendi gung-der-frau. Zugegriffen: 30. Apr. 2021.

Bauer, Werner T. 2014. Rechtsextreme und rechtspopulistische Parteien in Europa. Wien: ÖGPP. 
Bax, Daniel. 2017. Hauptsache, es geht gegen den Islam. Über die Rückkehr des Abendlandes. Indes Zeitschrift für Politik und Gesellschaft 2017(1):78-86.

Bebnowski, David. 2015. Die Alternative für Deutschland. Aufstieg und gesellschaftliche Repräsentanz einer rechten populistischen Partei. Wiesbaden: Springer VS.

Berberich, Frank, und Thilo Sarrazin. 2009. Klasse statt Masse. Von der Hauptstadt der Transferleistung zur Metropole der Eliten. Lettre International. Europas Kulturzeitung 86:197.

Betz, Hans-Georg. 2005. Against the system: radical right-wing populisms' challenge to liberal democracy. In Movements of exclusion: radical right-wing populism in the western world, Hrsg. Jens Rydgren, 25-40. New York: Nova Science.

Birsl, Ursula. 2017. Ähnlichkeiten und Unterschiede, Verflechtungen: die säkulare und religiöse Rechte in Deutschland. In Demokratie, Freiheit und Sicherheit. Festschrift zum 65. Geburtstag von Hans-Gerd Jaschke, Hrsg. Christoph Kopke, Wolfgang Kühnel, 105-130. Baden-Baden: Nomos.

Biskamp, Floris. 2018. Populism, religion and distorted communication. Public discourse, Islam, and the anti-Muslim mobilization of the Alternative for Germany. Zeitschrift für Religion, Gesellschaft und Politik 2(2):247-276.

Botsch, Gideon. 2020. Extremismusforscher hält AfD für rechtsextrem dominiert. https://rp-online.de/ politik/deutschland/extremismusforscher-haelt-afd-bundesweit-fuer-rechtsextrem-dominiert_aid51669297. Zugegriffen: 5. Mai 2021.

Brubaker, Rogers. 2017. Between nationalism and civilizationism: the European populist moment in comparative perspective. Ethnic and Racial Studies 40(8):1191-1226.

Bruckstein Çoruh, Almut Shulamit. 2010. Islam-Debatte in Deutschland. Die jüdisch-christliche Tradition ist eine Erfindung. https://de.qantara.de/inhalt/islam-debatte-in-deutschland-die-judisch-christlichetradition-ist-eine-erfindung. Zugegriffen: 22. Apr. 2021.

Bühl, Achim. 2017. Rassismus: Anatomie eines Machtverhältnisses. Bonn: Bundeszentrale für Politische Bildung.

Canovan, Margaret. 2002. Taking politics to the people: populism as the ideology of democracy. In Democracies and the populist challenge, Hrsg. Yves Mény, Yves Surel, 25-44. Basingstoke: Palgrave Macmillan.

Casanova, José. 2020. Transnationalism and religion: the European Union, from Christian-democratic project, to secular cosmopolitanism, to populist 'Christian' neo-nationalism. In Religion and neo-nationalism in Europe, Hrsg. Florian Höhne, Torsten Meireis, 29-48. Baden-Baden: Nomos.

Celik, Kazim, Oliver Decker, und Elmar Brähler. 2020. Rechtsextremismus für die breite Gesellschaft? Der Wandel der AfD-Wählerschaft von 2014 bis 2020. In Autoritäre Dynamiken. Alte Ressentiments - neue Radikalität. Leipziger Autoritarismus Studie 2020, Hrsg. Oliver Decker, Elmar Brähler, 149-177. Gießen: Psychosozial-Verlag.

Ceylan, Rauf. 2017. Islam und Muslime in Deutschland. Ein Überblick über die zweitgrößte Religionsgemeinschaft. Zeitschrift für Religion, Gesellschaft und Politik 1:75-88.

Corduwener, Pepijn. 2014. The populist conception of democracy beyond popular sovereignty. Journal of Contemporary European Research 10(4):423-437.

Cremer, Hendrik. 2019. Rassistische Hate Speech und Meinungsfreiheit. In Antimuslimischer Rassismus und Islamfeindlichkeit, Hrsg. Bülent Ucar, Wassilis Kassis, 103-124. Göttingen: V \& R unipress; Universitätsverlag Osnabrück.

Çağlar, Ayşe. 1990. The Prison House of Culture in the study of Turks in Germany. Sozialanthroplogische Arbeitspapiere 31:1-28.

Decker, Frank. 2006. Die populistische Herausforderung. Theoretische und ländervergleichende Perspektiven. In Populismus. Gefahr für die Demokratie oder nützliches Korrektiv?, Hrsg. Frank Decker, 9-32. Wiesbaden: VS.

Decker, Frank. 2011. Demokratischer Populismus und/oder populistische Demokratie? Bemerkungen zu einem schwierigen Verhältnis. In Populismus in der modernen Demokratie. Die Niederlande und Deutschland im Vergleich, Hrsg. Florian Hartleb, Friso Wielenga, 39-54. Münster: Waxmann.

Decker, Frank. 2013. Wenn die Populisten kommen. Beiträge zum Zustand der Demokratie und des Parteiensystems. Wiesbaden: Springer VS.

Decker, Frank. 2018. Autoritäre Wende? Rechtspopulismus und Rechtsextremismus in Europa. In Populismus und Politische Bildung, Hrsg. Sabine Manzel, Laura Möllers, 15-23. Frankfurt a. M.: Wochenschau Verlag.

Decker, Frank, und Marcel Lewandowsky. 2012. Die rechtspopulistische Parteienfamilie. In Parteienfamilien. Identitätsbestimmend oder nur noch Etikett?, Hrsg. Benjamin Höhne, Uwe Jun, 268-281. Opladen: Budrich. 
Decker, Oliver, Johannes Kiess, Julia Schuler, Barbara Handke, Gert Pickel, und Elmar Brähler. 2020. Die Leipziger Autoritarismus Studie 2020: Methode, Ergebnisse und Langzeitverlauf. In Autoritäre Dynamiken. Alte Ressentiments - neue Radikalität, Hrsg. Oliver Decker, Elmar Brähler, 27-87.

Dolezal, Martin, Laurenz Ennser-Jedenastik, Wolfgang C. Müller, und Anna Katharina Winkler. 2014. How parties compete for votes: a test of saliency theory. European Journal of Political Research 53:57-76.

Forlenza, Rosario. 2019. 'Abendland in Christian hands': Religion and populism in contemporary European politics. In Migration, gender and religion Populism and the crisis of democracy, Bd. 3, Hrsg. Gregor Fitzi, Jürgen Mackert, und Bryan S. Turner, 133-149. London: Routledge.

Foroutan, Naika. 2019. Die postmigrantische Gesellschaft: Ein Versprechen der pluralen Demokratie. Bielefeld: transcript.

Foroutan, Naika, Coşkun Canan, Frank Kalter, und Mara Simon. 2019. Ostmigrantische Analogien I: Konkurrenz um Anerkennung. Berlin: DeZIM-Institut.

Franzmann, Simon Tobias. 2014. Die Wahlprogrammatik der AfD in vergleichender Perspektive. Mitteilungen des Institutes für Internationales Parteienrecht und Parteienforschung an der Heinrich-HeineUniversität Düsseldorf 20:114-124.

Giebler, Heiko, Marcel Lewandowsky, und Aiko Wagner. 2015. Alles neu macht der Mai? Die Alternative für Deutschland (AfD) und die Europawahl 2014. In Die Europawahl 2014, Hrsg. Michael Kaeding, Niko Switek, 137-148. Wiesbaden: Springer.

Grabow, Karsten. 2016. PEGIDA and the Alternative für Deutschland: two sides of the same coin? European View 15:173-181.

Grimm, Marc, und Bodo Kahmann. 2017. Eine Partei im Spannungsfeld von Antisemitismus, Schuldabwehr und instrumenteller Israelsolidarität. In AfD \& FPÖ. Antisemitismus, völkischer Nationalismus und Geschlechterbilder, Hrsg. Stephan Grigat, 41-60. Baden-Baden: Nomos.

Hafez, Farid. 2019. Feindbild Islam: Über die Salonfähigkeit von Rassismus. Göttingen: Böhlau Verlag Wien.

Hambauer, Verena, und Anja Mays. 2018. Wer wählt die AfD? - Ein Vergleich der Sozialstruktur, politischen Einstellungen und Einstellungen zu Flüchtlingen zwischen AfD-WählerInnen und der WählerInnen der anderen Parteien. Zeitschrift für Vergleichende Politikwissenschaft 12:133-154.

Häusler, Alexander. 2015. AfD, Pegida \& Co. Die Formierung einer muslimfeindlichen rechten Bewegung. In Muslime in Deutschland. Historische Bestandsaufnahme, aktuelle Entwicklungen und zukünftige Forschungsfragen, Hrsg. Peter Antes, Rauf Ceylan, 59-76. Wiesbaden: Springer VS.

Häusler, Alexander. 2017. AfD, Pegida \& Co.: Die Formierung einer muslimfeindlichen rechten Bewegung. In Muslime in Deutschland. Historische Bestandsaufnahme, aktuelle Entwicklungen und zukünftige Forschungsfragen, Hrsg. Peter Antes, Rauf Ceylan, Naika Foroutan, und Andreas Zick, 59-74. Wiesbaden: Springer VS.

Häusler, Alexander. 2019. Antimuslimischer Populismus: Rechter Rassismus in neuem Gewand. Berlin: Aktion Courage e. V..

Häusler, Alexander, und Rainer Roeser. 2014. Rechtspopulismus in Europa und die rechtspopulistische Lücke in Deutschland. Erfurt: MOBIT.

Häusler, Alexander, und Rainer Roeser. 2015. Die rechten Mut-Bürger. Entstehung, Entwicklung, Personal und Positionen der Alternative für Deutschland. Hamburg: VSA.

Heinisch, Reinhard, und Oscar Mazzoleni. 2017. Analysing and explaining populism: bringing frame, actor and context back. In Political populism. A handbook, Hrsg. Reinhard Heinisch, Christina HoltzBacha, und Oscar Mazzoleni, 105-122. Baden-Baden: Nomos.

Heitmeyer, Wilhelm, und Anna Klein. 2012. Demokratie auf dem rechten Weg? Entwicklungen rechtspopulistischer Orientierungen und politischen Verhaltens in den letzten zehn Jahren. In Deutsche Zustände. Folge 10, Hrsg. Wilhelm Heitmeyer, 87-104. Berlin: Suhrkamp.

Hidalgo, Oliver. 2018. Religion, (Rechts-)Populismus und Demokratie - Versuch einer theoretischen Verhältnisbestimmung. Zeitschrift für Religion, Gesellschaft und Politik 2(2):167-192.

Hidalgo, Oliver, Philipp W. Hildmann, und Alexander Yendell. 2019. Religion und Rechtspopulismus. https://www.hss.de/download/publications/Argu_Kompakt_2019-3_Religion.pdf. Zugegriffen: 14. Jan. 2021. Argumentation Kompakt 3/2019.

Hierl, Katharina. 2012. Die Islamisierung der deutschen Integrationsdebatte: Zur Konstruktion kultureller Identitäten, Differenzen und Grenzziehungen im postkolonialen Diskurs. Berlin: LIT.

Hofrichter, Jürgen, und Oskar Niedermayer. 2016. Die Wählerschaft der AfD: Wer ist sie, woher kommt sie und wie weit rechts steht sie? Zeitschrift für Parlamentsfragen 2:267-284. 
Huber, Stefan, und Alexander Yendell. 2019. Does Religiosity Matter? Explaining right-wing extremist attitudes and the vote for Alternative for Germany (AfD). Religion and Society in Central and Eastern Europe 12(1):63-82.

Hund, Wulf D. 2018. Wie die Deutschen weiß wurden: Kleine (Heimat)Geschichte des Rassismus. Bonn: Bundeszentrale für Politische Bildung.

Huntington, Samuel P. 1996. Kampf der Kulturen: Die Neugestaltung der Weltpolitik im 21. Jahrhundert. München/Wien: Europa-Verl..

IKG (Institut für interdisziplinäre Konflikt- und Gewaltforschung). 2011. Presseinformation - Deutsche Zustände. Das entsicherte Jahrzehnt. http://www.uni-bielefeld.de/ikg/Handout_Fassung_Montag_ 1212.pdf. Zugegriffen: 13. Jan. 2021.

Jentsch, Ulli. 2016. Die „Lebensschutz“-Bewegung und die AfD. In Die Alternative für Deutschland. Programmatik, Entwicklung und politische Verortung, Hrsg. Alexander Häusler, 99-107. Wiesbaden: Springer VS.

Kampling, Rainer. 2012. Religiöses Vorurteil. In Vorurteile. Ursprünge, Formen, Bedeutung, Hrsg. Anton Pelinka, 147-168. Berlin: De Gruyter.

Karakayali, Juliane, und Paul Mecheril. 2018. Umkämpfte Krisen: Migrationsregime als Analyseperspektive migrationsgesellschaftlicher Gegenwart. In Postmigrantische Perspektiven. Ordnungssysteme, Repräsentationen, Kritik, Hrsg. Naika Foroutan, Juliane Karakayali, und Riem Spielhaus, 225-235.

Karakaşoğlu, Yasemin. 2009. Beschwörung und Vernachlässigung der Interkulturellen Bildung im „Integrationsland Deutschland“: Ein Essay. In Kulturen der Bildung, Beiträge zum 21. Kongress der Deutschen Gesellschaft für Erziehungswissenschaften. Schriftenreihe der Deutschen Gesellschaft für Erziehungswissenschaften (DGfE), Hrsg. Wolfgang Melzer, Rudolf Tippelt, 177-195. Opladen: Budrich.

Kemper, Andreas. 2020. Die reaktionäre Diskurskoalition. https://andreaskemper.org/2020/10/20/diereaktionaere-diskurskoalition/. Zugegriffen: 30. Apr. 2021.

Keskinkılıç, Ozan Zakariya. 2019. Die Islamdebatte gehört zu Deutschland: Rechtspopulismus und antimuslimischer Rassismus im (post-)kolonialen Kontext. Berlin: AphorismA.

Kiess, Johannes, Oliver Decker, Ayline Heller, und Elmar Brähler. 2020. Antisemitismus als antimodernes Ressentiment. Struktur und Verbreitung eines Weltbildes. In Autoritäre Dynamiken. Alte Ressentiments - neue Radikalität, Hrsg. Oliver Decker, Elmar Brähler, 211-248.

Korsch, Felix. 2016. „Natürliche Verbündete“? Die Pegida-Debatte in der AfD zwischen Anziehung und Ablehnung. In Die „Alternative für Deutschland“ - Entwicklung und politische Verortung, Hrsg. Alexander Häusler, 111-134. Wiesbaden: Springer VS.

Lang, Juliane. 2017. Feindbild Feminismus. Familien- und Geschlechterpolitik in der AfD. In AfD \& FPÖ. Antisemitismus, völkischer Nationalismus und Geschlechterbilder, Hrsg. Stephan Grigat, 61-78. Baden-Baden: Nomos.

Leibold, Jürgen, und Steffen Kühnel. 2003. Islamophobie. Sensible Aufmerksamkeit für spannungsreiche Anzeichen. In Deutsche Zustände, Folge 2, Hrsg. Wilhelm Heitmeyer. Frankfurt a.M.: Suhrkamp.

Lengfeld, Holger. 2018. Der „Kleine Mann“ und die AfD: Was steckt dahinter? Antwort an meine Kritiker. Kölner Zeitschrift für Soziologie und Sozialpsychologie 70:295-310.

Meyer, Thomas M., und Markus Wagner. 2013. Mainstream or niche? Vote-seeking incentives and the programmatic strategies of political parties. Comparative Political Studies 46(10):1246-1272.

Morgenstern, Christine. 2002. Rassismus - Konturen einer Ideologie: Einwanderung im politischen Diskurs der Bundesrepublik Deutschland. Hamburg: Argument.

Mouritsen, Per. 2006. The particular universalism of a Nordic civic nation: common values. State religion and Islam. In Danish political culture. In multiculturalism, muslim and citizenship: a European approach, Hrsg. Tariq Modood, Anna Triandafyllidou, und Ricard Zapata Barrero, 70-91. London: Routledge.

Mudde, Cas. 2004. The populist zeitgeist. Government and Opposition 39(4):541-563.

Müller, Wolfgang C., und Kaare Strøm. 2010. Political parties and hard choices. In Policy, office, or votes? How political parties in western Europe make hard decisions, Hrsg. Wolfgang C. Müller, Kaare Strøm, 1-35. Cambridge: Cambridge University Press.

Pautz, Hartwig. 2005. Die deutsche Leitkultur: Eine Identitätsdebatte; neue Rechte, Neorassismus und Normalisierungsbemühungen. Stuttgart: Ibidem.

Pesthy, Maria, Matthias Mader, und Harald Schoen. 2020. Why is the AfD so successful in eastern Germany? An analysis of the ideational foundations of the AfD vote in the 2017 federal election. Politische Vierteljahresschrift 62:69-91.

Pfahl-Traughber, Armin. 2019. Die AfD und der Rechtsextremismus. Eine Analyse aus politikwissenschaftlicher Perspektive. Wiesbaden: Springer VS. 
Pickel, Gert. 2018. Religion als Ressource für Rechtspopulismus? Zwischen Wahlverwandtschaften und Fremdzuschreibungen. Zeitschrift für Religion, Gesellschaft und Politik 2:277-312.

Pickel, Gert, Susanne Pickel, und Alexander Yendell. 2020. Zersetzungspotenziale einer demokratischen politischen Kultur: Verschwörungstheorien und erodierender gesellschaftlicher Zusammenhalt. In Autoritäre Dynamiken. Alte Ressentiments - neue Radikalität, Hrsg. Oliver Decker, Elmar Brähler, 89-118. Gießen: Psychosozial-Verlag.

Rensmann, Lars. 2020. Die Mobilisierung des Ressentiments. Zur Analyse des Antisemitismus in der AfD. In Prekärer Zusammenhalt. Die Bedrohung des demokratischen Miteinanders in Deutschland, Hrsg. Ayline Heller, Oliver Decker, und Elmar Brähler, 309-342. Gießen: Psychosozial-Verlag.

Roy, Olivier. 2016. Beyond populism: the conservative right, the courts, the churches and the concept of a Christian Europe. In Saving the people: how populists hijack religion, Hrsg. Nadia Marzouki, Duncan McDonnell, und Olivier Roy, 185-202. London: Hurst and Publishers.

Rydgren, Jens. 2004. The populist challenge. Political protest and ethno-nationalist mobilization in France. New York, Oxford: Berghahn Books.

Rydgren, Jens. 2005. Is extreme right-wing populism contagious? Explaining the emergence of a new party family. European Journal of Political Research 44:413-437.

Said, Edward W. 2017. Orientalismus. Frankfurt am Main: S. Fischer.

Salzborn, Samuel. 2018. Populismus als Herausforderung für die beschleunigte Demokratie im 21. Jahrhundert. In Populismus und Politische Bildung. Hrsg. Sabine Manzel, Laura Möllers, 24-31. Frankfurt a. M.: Wochenschau.

Salzborn, Samuel. 2019. Antisemitismus in der „Alternative für Deutschland“. In Antisemitimus seit 9/11. Ereignisse, Debatten, Kontroversen, Hrsg. Samuel Salzborn, 197-216. Baden-Baden: Nomos.

Sarrazin, Thilo. 2010. Deutschland schafft sich ab. Wie wir unser Land aufs Spiel setzen. München: Deutsche Verlags-Anstalt.

Schönfelder, Sven. 2013. Europäischer Rechtspopulismus. Themen und Strategien. In Die neuen Rechten in Europa. Zwischen Neoliberalismus und Rassismus. Hrsg. Peter Bathke, Anke Hoffstadt, 96-111. Köln: Papyrossa.

Schwarz, Karolin. 2020. Hasskrieger. Der neue globale Rechtsextremismus. Freiburg i. Br.: Herder.

Shooman, Yasemin. 2014. „... weil ihre Kultur so ist“: Narrative des antimuslimischen Rassismus. Bielefeld: transcript.

Siri, Jasmin. 2016. Geschlechterpolitische Positionen der Partei Alternative für Deutschland. In Die Alternative für Deutschland. Programmatik, Entwicklung und politische Verortung, Hrsg. Alexander Häusler, 69-80. Wiesbaden: Springer VS.

Spielhaus, Riem. 2013. Vom Migranten zum Muslim und wieder zurück: Die Vermengung von Integrations- und Islamthemen in Medien, Politik und Forschung. In Islam und die deutsche Gesellschaft, Hrsg. Dirk Halm, Hendrik Meyer, 169-194. Wiesbaden: Springer VS.

Spielhaus, Riem. 2018. Zwischen Migrantisierung von Muslimen und Islamisierung von Migranten. In Postmigrantische Perspektiven. Ordnungssysteme, Repräsentationen, Kritik, Hrsg. Naika Foroutan, Julia Karakayali, und Riem Spielhaus, 129-143. Bonn: Bundeszentrale für politische Bildung.

Spier, Tim. 2010. Modernisierungsverlierer? Die Wählerschaft rechtspopulistischer Parteien in Westeuropa. Wiesbaden: VS.

Stanley, Ben. 2008. The thin ideology of populism. Journal of Political Ideologies 13(1):95-110.

Taggart, Paul. 2002. Populism and the pathology of represantative politics. In Democracies and the populist challenge, Hrsg. Yves Mény, Yves Surel, 62-80. Basingstoke: Palgrave Macmillan.

Taggart, Paul. 2004. Populism and the representative politics in contemporary Europe. Journal of Political Ideologies 9(3):269-288.

Tezcan, Levent. 2012. Das muslimische Subjekt. Verfangen im Dialog der Deutschen Islam Konferenz. Konstanz: University Press.

Thieme, Tim. 2019. Dialog oder Ausgrenzung - Ist die AfD eine rechtsextreme Partei? http://www. bpb.de/politik/extremismus/rechtspopulismus/284482/dialog-oder-ausgrenzung-ist-die-afd-einerechtsextreme-partei. Zugegriffen: 23. Jan. 2020.

Tiesler, Nina Clara. 2006. Muslime in Europa: Religion und Identitätspolitiken unter veränderten gesellschaftlichen Verhältnissen. Berlin: Lit-Verl.

Volkov, Shulamit. 2000. Antisemitismus als kultureller Code. München: C. H. Beck.

Weber, Karl. 2017. Die globale Flüchtlingskrise als Herausforderung für die kirchliche Entwicklungszusammenarbeit. In Verändert die europäische Flüchtlingskrise die Entwicklungszusammenarbeit?, Hrsg. Hartmut Sangmeister, Heike Wagner, 79-88. Baden-Baden: Nomos.

Wildt, Michael. 2017. Volk, Volksgemeinschaft, AfD. Bonn: bpb. 
Wodak, Ruth. 2018. Vom Rande in die Mitte - ,Schamlose Normalisierung“. Politische Vierteljahresschrift 59:323-335.

Yalçın, Cem Serkan. 2019. Rechtspopulismus: Vorurteil aus sozialpsychologischer Sicht. In Antimuslimischer Rassismus und Islamfeindlichkeit, Hrsg. Bülent Ucar, Wassilis Kassis, 309-335. Göttingen: V \& R unipress; Universitätsverlag Osnabrück.

\section{Quellen}

AfD BB. 2019. Landtagswahlprogramm für Brandenburg 2019. Hol dir dein Land zurück. https://afdbrandenburg.de/wp-content/uploads/2019/06/Wahlprogramm_Brandenburg_2019_ohne_kapitelbild er_kommentare_acc2144-01-06-19-final.pdf. Zugegriffen: 15. Jan. 2021.

AfD BE. 2017. Facebook. https://www.facebook.com/afdberlin/posts/-israels-flagge-brennt-schonwieder-seit-freitag-gibt-es-in-berlin-eine-reihe-vo/815841935264607. Zugegriffen: 15. Jan. 2021.

AfD BE. 2018. Facebook. https://www.facebook.com/afdberlin/posts/-wer-sich-solidarisch-mit-israelzeigt-hat-in-berlin-einen-schweren-stand-polize/1001228613392604. Zugegriffen: 15. Jan. 2021.

AfD BW. 2016. Landtagswahlprogramm 2016 der AfD Baden-Württemberg. Für unser Land - für unsere Werte. https://afd-bw.de/afd-bw/wahlprogramme/landtagswahlprogramm_afd_2016_1.pdf. Zugegriffen: 15. Jan. 2021.

AfD BW. 2018. Facebook. https://www.facebook.com/AfD.BW/posts/1838211836198677. Zugegriffen: 15. Jan. 2021.

AfD BY. 2018. Bayern. Aber sicher! Wahlprogramm Landtagswahl Bayern 2018. https://www.afdbayern. de/wahlen-2018/wahlprogramm-landtagswahl-2018. Zugegriffen: 15. Jan. 2021.

AfD HB. 2019. Wahlprogramm der AfD Hamburg für die Bürgerschaftswahl 2020. https://afd-hamburg. de/wp-content/uploads/2020/01/Bu\%CC\%88rgerschaftswahlprogramm-2020-der-AfD-Hamburg. pdf. Zugegriffen: 15. Jan. 2021.

AfD NI. 2017. Niedersachsen. Unsere Heimat. Unsere Zukunft. Landesprogramm des Landesverbandes Niedersachsen der Alternative für Deutschland. https://afd-niedersachsen.de/wp-content/uploads/ 2020/01/AFD_2017-05-07_Landesprogramm-. Zugegriffen: 15. Jan. 2021.

AfD NRW. 2017. Für unsere Familien und unsere Heimat. Wahlprogramm der AfD für die Bürger Nordrhein-Westfalens. https://cdn.afd.tools/sites/2/2017/03/24145045/LWP_Komplettprogramm_AK9_ RZ_Low.pdf. Zugegriffen: 15. Jan. 2021.

AfD SN. 2016. „Die Stimme der Bürger - Unser Programm!“ Wahlprogramm zur Landtagswahl am 13. März 2016. „Wir für unsere Heimat““. http://ltw16.sachsen-anhalt-waehlt.de/fileadmin/LTW2016/ Wahlprogramme/wahlprogramm_afd.pdf. Zugegriffen: 15. Jan. 2021.

AfD SN. 2019. Trau dich Sachsen. Regierungsprogramm der Alternative für Deutschland zur Landtagswahl Sachsen 2019. https://www.afdsachsen.de/wp-content/uploads/2020/07/RWP_190618.pdf. Zugegriffen: 15. Jan. 2021.

AfD TH. 2019. Meine Heimat, Mein Thüringen. Wahlprogramm der Alternative für Deutschland für die Landtagswahl in Thüringen 2019. https://cdn.afd.tools/wp-content/uploads/sites/178/2019/09/ Wahlprogramm_AfD-Thu\%CC\%88ringen_2019_Online-Fassung-final_gesichert.pdf. Zugegriffen: 15. Jan. 2021.

AfD-thl. 2017. Der Islam. Fakten und Argumente. https://afd-thl.de/download/16738. Zugegriffen: 15. Jan. 2021.

AfD. 2013. Wahlprogramm. Parteitagsbeschluss vom 14.04.2013. Manifesto Project Data Dashboard. https://visuals.manifesto-project.wzb.eu/mpdb-shiny/cmp_dashboard_dataset/. Zugegriffen: 23. Jan. 2020.

AfD. 2016. Programm für Deutschland. Das Grundsatzprogramm der Alternative für Deutschland. https:// www.afd.de/wp-content/uploads/sites/111/2018/01/Programm_AfD_Online-PDF_150616.pdf. Zugegriffen: 6. Jan. 2021.

AfD. 2017. Programm für Deutschland. Wahlprogramm der Alternative für Deutschland für die Wahl zum Deutschen Bundestag am 24. September 2017. https://www.afd.de/wp-content/uploads/sites/ 111/2017/06/2017-06-01_AfD-Bundestagswahlprogramm_Onlinefassung.pdf. Zugegriffen: 6. Jan. 2021.

Balzli, Beat, und Matthias Kamann. 2016. Petry will den Begriff 'völkisch’ positiv besetzen. https://www. welt.de/politik/deutschland/article158049092/Petry-will-den-Begriff-voelkisch-positiv-besetzen. html. Zugegriffen: 20. Apr. 2021. 
Bender, Justus, und Rüdiger Soldt. 2016. Im Eiferer-Modus gegen Juden. https://www.faz.net/aktuell/ politik/inland/antisemitische-thesen-von-afd-abgeordneten-gedeon-14268327.html. Zugegriffen: 20.09.2021.

Bündnis 90/Die Grünen, und Sozialdemokratische Partei Deutschlands. 1998. Aufbruch und Erneuerung. Deutschlands Weg ins 21. Jahrhundert. Koalitionsvereinbarung zwischen der Sozialdemokratischen Partei Deutschlands und Bündnis 90/Die Grünen. Bonn: C.H. Beck.

CDU. 2007. Freiheit und Sicherheit. Grundsätze für Deutschland. https://archiv.cdu.de/grundsatzprogramm2007. Zugegriffen: 30. Apr. 2021.

CSU. 2016. Die Ordnung. Grundsatzprogramm der Christlich-Sozialen Union. https://www.csu.de/ common/download/Grundsatzprogramm-Beschluss-Parteitag.pdf. Zugegriffen: 30. Apr. 2021.

Curio, Gottfried. 2018. „Rede von Gottfried Curio in Kirchhain“, Video vom 04.10.2018. https://www. youtube.com/watch?v=RZo3b1xU6JQ. Zugegriffen: 15. Jan. 2021.

DLF. 2017. „Denkmal der Schande“ Empörung über Höckes Äußerungen zum Holocaust-Mahnmal. https://www.deutschlandfunk.de/denkmal-der-schande-empoerung-ueber-hoeckes-aeusserungen. 1947.de.html?drn:news_id=700846. Zugegriffen: 15. Jan. 2021.

Gauland, Alexander. 2016. Hitler hat den Deutschen das Rückgrat gebrochen (Interview). https://www. zeit.de/2016/17/alexander-gauland-afd-cdu-konservatismus. Zugegriffen: 20.09.2021.

Gauland, Alexander. 2018. „Der Schutz Israels beginnt am Brandenburger Tor! - Dr. Alexander Gauland - AfD-Fraktion im Bundestag“, Video vom 26.04.2018. https://www.youtube.com/watch? v=Y5bZhqMSpdc. Zugegriffen: 15. Jan. 2021.

Gensing, Patrick. 2015. Die AfD und die ,Volksgemeinschaft‘. https://hajofunke.wordpress.com/2015/12/ 29/tagesschau-die-afd-und-die-volksgemeinschaft. Zugegriffen: 20. Apr. 2021.

Kemper, Andreas. 2016. Geschlechter- und familienpolitische Positionen der AfD. https://library.fes.de/ pdf-files/dialog/10641-20140414.pdf. Zugegriffen: 30. Apr. 2021.

Lucke, Bernd. 2013. Die zehn Thesen zum Islam. http://www.pi-news.net/2013/11/10-thesen-von-afdchef-bernd-lucke-zum-islam. Zugegriffen: 15. Jan. 2021.

Mascolo, Georg, Sebastian Pittelkow, und Katja Riedel. 2019. Verfassungsschutz: Beobachtung der AfD rückt näher. https://www.tagesschau.de/investigativ/ndr-wdr/afd-verfassungsschutz-157.html. Zugegriffen: 20. Apr. 2021.

Neuerer, Dietmar. 2017. Alle gegen die AfD. https://www.handelsblatt.com/politik/deutschland/bundestags wahl/alle-schlagzeilen/bundestagswahl-alle-gegen-die-afd/20343226-all.html. Zugegriffen: 14. Jan. 2021.

Saure, Hans-Wilhelm, und Anton Maegerle. 2016. Skandal um antisemitisches Buch von Wolfgang Gedeon. https://www.bild.de/politik/inland/alternative-fuer-deutschland/antisemitisches-buch-46065860. bild.html. Zugegriffen: 20.09.2021.

Siemens, Ansgar, Severin Weiland, und Wolf Wiedmann-Schmidt. 2020. Geheimdienstchef Haldenwang. Der Höcke-Jäger. https://www.spiegel.de/politik/verfassungsschutz-beobachtet-afd-fluegel-thomashaldenwang-der-hoecke-jaeger-a-00000000-0002-0001-0000-000169988549. Zugegriffen: 20. Apr. 2021.

Spiegel online. 2016. AfD will noch nicht regieren - jedenfalls Teile von ihr jetzt noch nicht. https:// www.spiegel.de/politik/deutschland/afd-will-noch-nicht-regieren-a-1088982.html. Zugegriffen: 13. Jan. 2021.

Weidel, Alice. 2017. Alice Weidel im Exklusiv-Interview: „Die AfD ist die einzige echte Schutzmacht für Schwule und Lesben in Deutschland“. https://www.afd.de/alice-weidel-im-exklusiv-interview-dieafd-ist-die-einzige-echte-schutzmacht-fuer-schwule-und-lesben-in-deutschland. Zugegriffen: 15. Jan. 2021.

Weidel, Alice. 2018. „Generalaussprache zur Regierungspolitik“. Video vom 16.05.2018. https://www. youtube.com/watch?v=Vc8Ra3FmE2Y. Zugegriffen: 15. Jan. 2021.

Weiland, Severin. 2016. Anti-Islam-Partei - wieder so ein Schlagwort. https://www.spiegel.de/politik/ deutschland/afd-chef-joerg-meuthen-anti-islam-partei-wieder-so-ein-schlagwort-a-1089575.html. Zugegriffen: 15. Jan. 2021. 